\title{
- Statistical Development of a Duty Cycle for Plug-in Vehicles in a North American Urban Setting Using Fleet Information
}

\author{
Soheil Shahidinejad, Eric Bibeau, and Shaahin Filizadeh, Member, IEEE
}

\begin{abstract}
5 Abstract-Development of a daily duty cycle based on real6 world driving behavior and parking times is a critical requirement 7 in the optimal design of power-train components of a plug-in 8 vehicle. Standard driving cycles cannot completely emulate the 9 real-world power demand of a vehicle and its downtimes in par10 ticular. To address these shortcomings, a large database of one 11 year of measured data collected from a fleet of $\mathbf{7 6}$ cars in the 12 city of Winnipeg, MB, Canada is obtained and is then used to 13 develop a new duty cycle. This paper describes a methodology 14 for statistical analysis of the fleet data, including while a vehicle 15 is parked. Due to the intrinsic differences in vehicle usage profiles 16 during weekdays and weekends, two 24 -h duty cycles with suitable 17 windows of opportunity for charging are developed for weekday 18 and weekend driving patterns. The uniqueness of the proposed 19 statistical methodology and the resulting duty cycles contribute to 20 addressing the present shortcomings of standard driving cycles.
\end{abstract}

21 Index Terms-Battery storage, driving cycle, duty cycle, electric 22 vehicle, plug-in hybrid vehicles, renewable energy.

\section{INTRODUCTION}

24 LECTRIFICATION of transportation for light-duty vehi25 cles is a prominent step toward sustainable transportation 26 [1], [3]. It can also contribute to efficient integration and use 27 of existing and emerging renewable energy resources. Plug-in 28 vehicles (i.e., pure electric or plug-in hybrids) have a strong 29 potential to reduce petroleum consumption by shifting energy 30 demand away from fossil fuels to electrical energy that is 31 domestically produced using renewable sources. A plug-in 32 vehicle allows its battery storage to recharge via connection 33 to a utility grid while the vehicle is parked. Therefore, it 34 covers a wide range of vehicles using electricity as a source of 35 propulsion either partially, such as in a plug-in hybrid electric 36 vehicle (PHEV), or entirely, such as in a battery electric vehicle 37 (BEV). When used in conjunction with a distributed high38 capacity-storage electric utility, it will also help accommodate

Manuscript received April 13, 2010; revised June 21, 2010; accepted July 16, 2010. This work was supported by the AUTO21 Network of Centers of Excellence under Project DF302-DBS. The review of this paper was coordinated by Dr. A. Khaligh.

S. Shahidinejad and E. Bibeau are with the Department of Mechanical and Manufacturing Engineering, University of Manitoba, Winnipeg, MB R3T 5V6, Canada (e-mail: shahidin@cc.umanitoba.ca; bibeauel@cc.umanitoba.ca).

S. Filizadeh is with the Department of Electrical and Computer Engineering, University of Manitoba, Winnipeg, MB R3T 5V6, Canada (e-mail: sfilizad@ee.umanitoba.ca).

Color versions of one or more of the figures in this paper are available online at http://ieeexplore.ieee.org.

Digital Object Identifier 10.1109/TVT.2010.2061243 the variable and unpredictable nature of renewable sources. It 39 is envisioned that by increasing the share of renewable energies 40 for electric power generation and optimizing rechargeable en- 41 ergy storage battery units in plug-in vehicles, major concerns 42 with regard to peak oil, greenhouse gases leading to climate 43 change, energy security, and emissions, can be simultaneously 44 addressed [4]. Due to high cost and large weight per unit energy 45 capacity of current battery cells, the technology pathway for 46 PHEVs to lower the battery size and cost includes providing 47 additional daily charging opportunities during periods when the 48 vehicles are parked and opportunities for charging exist [3]-[7]. 49

Complete assessment of the potential power and energy 50 demand in plug-in vehicles is required to simulate and optimize 51 their energy-storage systems [8]. Optimal sizing of the electric 52 drive-train components, choice of battery chemistry and storage 53 size, development of controllers tuned and optimized to vehicle 54 driving patterns, as well as realistic opportunity charging sce- 55 narios, all require detailed information on the vehicle's usage 56 profile. Obviously, recharging scenarios and grid impacts can be 57 better analyzed with detailed information on parking durations, 58 as well as time and location of parking events in drivers' 59 daily routines. Real-world driving patterns provide insight into 60 speed and acceleration characteristics. However, only stan- 61 dard driving schedules conducted on dynamometers or well- 62 documented tracks have been accepted as a systematic approach 63 to mimic real-life situations. Standard certification driving 64 cycles such as the Urban Dynamometer Driving Schedule 65 (UDDS) or Highway Fuel Economy Test have been convention- 66 ally used in conjunction with controlled chassis dynamometer 67 testing to represent average driving behavior of the drivers in 68 fuel economy and emission certification of the vehicles.

It is important to note that the standard certification cycles are 70 still unable to handle extreme acceleration or deceleration rates 71 that fall beyond capabilities of laboratory equipment and are 72 bound to limited cycle durations, i.e., usually less than $20 \mathrm{~min}, 73$ to keep test costs low [5], [12]. For instance, the FTP72 driving 74 cycle, which dates back to early 1970s, was primarily developed 75 to measure exhaust emissions of typical light-duty vehicle 76 operations in the Los Angeles urban area [13]. To address the 77 shortcomings of the FTP72 cycle in representing more aggres- 78 sive speeds and accelerations, a Unified Cycle was developed 79 in 1992 based on collected data, known as LA92. However, 80 there are still many concerns about the problems inherent in the 81 existing driving cycles, which lead to underestimation of cruise, 82 acceleration, or stop-and-go activities in different brackets of 83 
84 velocities [14]. It is therefore concluded that such cycles cannot 85 completely emulate the real-world daily power demand of a 86 vehicle. More importantly, they do not provide information 87 on parking times as opportunities for charging in the case of 88 emerging plug-in vehicles. In addition, it is important to base 89 duty cycles on larger data sets to reduce statistical errors.

90 An extensive literature survey conducted revealed no refer91 ence to the development of a daily driving cycle, taking into 92 account power and energy demands as major requirements for 93 representing the real-world data. Most studies have focused on 94 developing urban driving cycles using snippets extracted from 95 recorded speed-time traces to estimate vehicular emissions and 96 fuel consumption in different cosmopolitan areas [15], [19]. 97 The objective of these studies is to represent the driving infor98 mation in a collected data set using a single driving cycle. Some 99 other studies discuss the effectiveness of the methodologies 100 used in developing driving cycles to represent the inherent char101 acteristics of driving behavior in the collected data [20], [21].

102 A few recent studies exist that have assessed the performance 103 of hybrid electric vehicles (HEVs) in real-world operation. 104 For example, data collected for a fleet in the St. Louis, MO, 105 metropolitan area were used in the simulation of energy usage 106 in a PHEV, but no single driving pattern was extracted from 107 the collected data [22]. Fuzzy logic pattern recognition tech108 niques have also been used to perform driving and duty cycle 109 analyses on data collected for a fleet of HEVs [23]. Another 110 effort to modify standard cycles for better representing real111 world behavior introduced a driver model in connection with 112 European standard cycles into simulations [24]. A methodology 113 that generates a driving cycle has also been reported based 114 on the assumption of constant acceleration and deceleration 115 rates, along with consideration of the speed limits in different 116 road segments in representative areas [25]. It can therefore be 117 concluded that there does not exist a single widely accepted 118 duty cycle in the literature to appropriately represent typical 119 daily activities of the vehicles and to address the energy and 120 power demands of the PHEVs and BEVs.

121 The study presented in this paper addresses the gap in the 122 literature by developing a statistical methodology and con123 structing the needed duty cycles based on a database of over 12444 million Global Positioning System (GPS) data points 125 recorded over the course of one year in Winnipeg, MB, Canada. 126 Depending on the context, the terms "driving cycle" and 127 "duty cycle" may convey different meanings. In this paper, 128 a "driving cycle" refers to a history of daily driving periods 129 represented by a speed-versus-time curve. A "duty cycle" refers 130 to a profile of daily usage of power by the vehicle, which is 131 typically represented by a 24-h history of driving and parking 132 events. Note that, in the case of an HEV, only a driving cycle 133 is sufficient to calculate vehicle's power demand, whereas in 134 the case of a plug-in vehicle, parking times also become vital 135 as they may be used for charging from the electric grid, and 136 therefore, they should be included in the daily profile. Parking 137 times are also important to utilities servicing jurisdictions with 138 large vehicular loads, as they can be used to predict and control 139 the load on the grid.

140 The objective of the present study is to first develop a 141 new driving cycle most closely mirroring the characteristics of

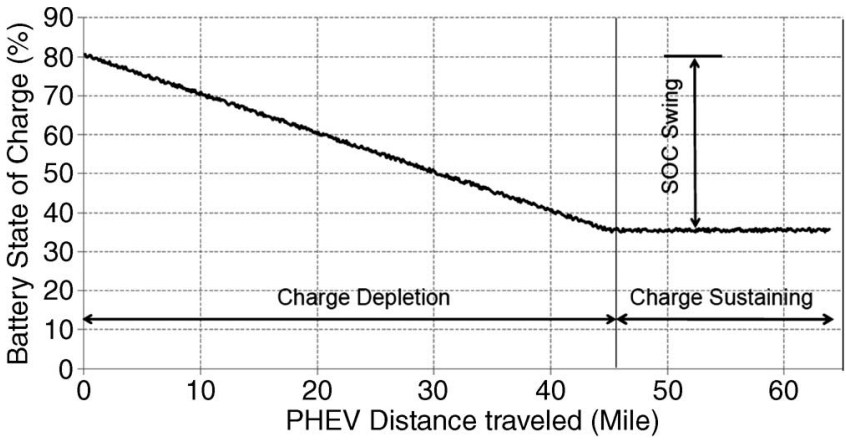

Fig. 1. Typical variation in the SOC of a PHEV battery.

urban driving, including real-world energy and power demands. 142 Once this is achieved, this study aims to incorporate a pattern 143 representing most probable downtimes of the vehicles to charge 144 during their daily usage profile. A comprehensive daily duty 145 cycle is a crucial component for optimal design of plug-in 146 vehicular drive-trains. This study improves the conventional 147 methods of driving cycle generation [26], [28]. The present 148 study also establishes a set of performance measures required to 149 assess a driving cycle suited for electric/plug-in hybrid vehicles. 150 The methodology used in the precedent study for simulation 151 of a plug-in vehicle is enhanced by including 25 parameters 152 to characterize different velocity brackets in a driving cycle 153 [29]. The uniqueness of this study is proposing a methodol- 154 ogy that addresses the particular requirements associated with 155 the design of plug-in vehicles in construction of a 24-h duty 156 cycle.

Following the introduction, in Section II, duty cycle re- 158 quirements to be fulfilled for enhanced simulation and opti- 159 mization of plug-in vehicles are discussed. Data collection, 160 driving cycle generation, and characterization are described 161 in Section III. Parking data analysis for weekdays and week- 162 ends is presented in Section IV. The resulting 24-h duty 163 cycles are given in Section V, and driving characteristics 164 are critically compared with those of the standard cycle for 165 urban driving, i.e., the UDDS. Conclusions are presented 166 in Section VI.

\section{Duty Cycle ReQuirements for Plug-IN Vehicles 168}

The total distance that a plug-in vehicle can electrically 169 drive is an important measure for the vehicle's performance 170 assessment. For instance, PHEVs are usually classified ac- 171 cording to their all-electric range (AER), which is defined as 172 the total miles electrically driven after a full recharge before 173 the engine turns on for the first time [30]. A fully recharged 174 PHEV operates in charge-depleting mode until the battery is 175 depleted to a target state-of-charge (SOC), at which point, the 176 vehicle switches to charge-sustaining mode, using the internal 177 combustion engine to maintain the target SOC [3]. Fig. 1 shows 178 the typical variation in the SOC of the battery in the operating 179 modes of a PHEV.

Obviously, the instantaneous power demand resulting from 181 the driving style of the driver is critical in the definition of the 182 AER. The standard cycle UDDS is usually used to measure 183 
TABLE I

CHARACTERIZING PARAMETERS AND THEIR VALUES

\begin{tabular}{|c|c|c|c|c|c|}
\hline & \multirow{2}{*}{ Parameter } & \multicolumn{2}{|c|}{ Database average } & \multicolumn{2}{|c|}{ Final duty cycles } \\
\hline & & weekday & weekend & Weekday & weekend \\
\hline 1 & Average speed of the entire driving cycle in $\mathrm{km} / \mathrm{h}$ & 31.4 & 35.3 & 32.6 & 35.0 \\
\hline 2 & Average running speed in $\mathrm{km} / \mathrm{h}$ & 39.8 & 43.9 & 40.3 & 43.4 \\
\hline 3 & Total daily distance traveled in $\mathrm{km}$ & 32.2 & 33.9 & 31.6 & 35.2 \\
\hline 4 & Average acceleration of all acceleration phases in $\mathrm{m} / \mathrm{s} 2$ & 0.6 & 0.6 & 0.55 & 0.59 \\
\hline 5 & Average deceleration of all deceleration phases in $\mathrm{m} / \mathrm{s} 2$ & -0.6 & -0.6 & -0.59 & -0.6 \\
\hline 6 & Average number of change in acceleration rate $(+/-)$ in one driving period & 6.5 & 7.2 & 7.83 & 7.9 \\
\hline 7 & Average daily power demand in $\mathrm{kW}$ & 7.9 & 8.3 & 8.92 & 9.3 \\
\hline 8 & Maximum power demand in $\mathrm{kW}$ & 59.3 & 72.2 & 49.45 & 60.0 \\
\hline 9 & Total daily energy demand in $\mathrm{Mj}$ & 17.3 & 17.7 & 16.41 & 18.2 \\
\hline 10 & Average daily breaking power in $\mathrm{kW}$ & -5.3 & -5.4 & -5.47 & -6.0 \\
\hline 11 & Root mean square of acceleration in $\mathrm{m} / \mathrm{s} 2$ & 0.8 & 0.8 & 0.66 & 0.69 \\
\hline 12 & Average length of a driving period in $\mathrm{km}$ & 0.9 & 1.2 & 0.75 & 1.03 \\
\hline 13 & Time percentage of Idling (zero velocity) in \% & 21.8 & 20.7 & 19.1 & 19.31 \\
\hline 14 & Time percentage of acceleration: acceleration $>0.1 \mathrm{~m} / \mathrm{s} 2$ in $\%$ & 31.4 & 31.3 & 36.5 & 35.1 \\
\hline 15 & Time percentage of Cruising (acceleration $[-0.1,0.1] \mathrm{m} / \mathrm{s} 2$, speed $>5 \mathrm{~m} / \mathrm{s}$ ) in $\%$ & 14.9 & 16.7 & 9.8 & 10.5 \\
\hline 16 & Time percentage of deceleration: acceleration $<-0.1 \mathrm{~m} / \mathrm{s} 2$ in $\%$ & 31.2 & 30.9 & 34.4 & 34.9 \\
\hline 17 & Time percentage of creeping (acceleration $[-0.1,0.1] \mathrm{m} / \mathrm{s} 2$, speed $<5 \mathrm{~m} / \mathrm{s}$ ) in $\%$ & 0.6 & 0.5 & 0.32 & 0.14 \\
\hline 18 & $\begin{array}{l}\text { Time percentage of driving at very low speed bracket } \\
{\left[\begin{array}{ll}0 & 20\end{array}\right] \mathrm{km} / \mathrm{h} \text { in } \%}\end{array}$ & 94.5 & 90.6 & 85.3 & 90.5 \\
\hline 19 & Average speed in very low speed bracket $\left[\begin{array}{lll}0 & 20\end{array}\right] \mathrm{km} / \mathrm{h}$ & 6.6 & 10.3 & 7.3 & 6.5 \\
\hline 20 & $\begin{array}{l}\text { Time percentage of driving at low speed bracket } \\
{[2050] \mathrm{km} / \mathrm{h} \text { in } \%}\end{array}$ & 58.9 & 59.6 & 62.0 & 61.0 \\
\hline 21 & Average speed in low speed bracket $\left[\begin{array}{ll}20 & 50\end{array}\right] \mathrm{km} / \mathrm{h}$ & 18.9 & 20.8 & 19.2 & 28.1 \\
\hline 22 & Time percentage of driving at moderate speed bracket $\left[\begin{array}{ll}50 & 80\end{array}\right] \mathrm{km} / \mathrm{h}$ in $\%$ & 36.7 & 36.9 & 37.4 & 36.4 \\
\hline 23 & Average speed in moderate speed bracket $\left[\begin{array}{ll}50 & 80\end{array}\right] \mathrm{km} / \mathrm{h}$ & 32.9 & 30.2 & 34.9 & 29.0 \\
\hline 24 & $\begin{array}{l}\text { Time percentage of driving at high speed bracket } \\
{\left[\begin{array}{ll}80 & 100\end{array}\right] \mathrm{km} / \mathrm{h} \text { in } \%}\end{array}$ & 9.9 & 10.0 & 8.9 & 10.6 \\
\hline 25 & Average speed in high speed bracket $\left[\begin{array}{lll}80 & 100\end{array}\right] \mathrm{km} / \mathrm{h}$ & 16.3 & 15.0 & 16.9 & 15.1 \\
\hline
\end{tabular}

184 the AER for classifying PHEVs. Therefore, a PHEV $x$ expected 185 to travel $x$ miles on battery, in the real world, would perform 186 quite differently, depending on the driving habits of the driver. 187 The mentioned difficulty in providing a realistic performance 188 measure to the end users is also valid in the case of an HEV. 189 The dominant mode of operation in an HEV battery is charge 190 sustaining, and its fuel efficiency is characterized by a higher 191 mile per gallon rate when compared with conventional vehicles. 192 Again, using the standard cycle is misleading as, in reality, fuel 193 consumption would be higher, depending on the aggressiveness 194 of the driver.

195 In this study, real-world driving data are collected over a 196 sufficiently long period to reduce statistical errors. In addition, 197 the characterizing parameters of the driving cycles used to 198 generate the standard driving cycles are extended to cover 199 average driver's daily energy demand and maximum power 200 demand (positive in acceleration and negative in deceleration) 201 for light-duty vehicles (with a dominant share in urban traffic). 202 The information on parking events such as the frequency of 203 occurrence and the ratio of parking to driving times in an 204 average daily driving profile is also included to construct an 205 average 24 -h duty cycle.

206 The characterizing parameters considered in this paper are 207 those defining power requirements at different driving modes, 208 as listed in Table I. Except for its considerably cold winter 209 temperatures, Winnipeg, with a population of 700 000, is a 210 typical North American urban setting where driving culture, 211 population, and traffic behavior are similar to many other cities 212 across the United States and Canada. Although the particular duty cycles developed in this study may be applied to many 213 other similarly populated cities, the methodology described in 214 the next section is general and can be used to develop duty 215 cycles for any other urban area of interest.

\section{Methodology of Development OF A DRIVING CYCLE}

The proposed methodology comprises two stages: 1) de- 219 veloping a driving cycle based on a large set of data points 220 collected and 2) incorporating results of a statistical analysis 221 on daily parking times to construct a 24-h duty cycle. An 222 enhanced statistical approach is developed using 25 parame- 223 ters to characterize the driving cycle by selecting candidates 224 from the database that have the closest match to the average 225 of the parameters. The candidate cycle is then incrementally 226 enhanced by replacing its microtrips with those extracted from 227 the same traffic group to minimize a figure of merit defined 228 based on average values of the characterizing parameters. The 229 processed parking data are then categorized in various groups 230 and included in the daily usage profile.

There are two general methodologies to develop a driving 232 cycle. One is based on creating a pool of trip segments extracted 233 from recorded speed-time traces of vehicles, followed by cat- 234 egorizing them into several driving modes and finally patching 235 snippets selected based on desired selection criteria together 236 to develop a representative driving cycle with a predetermined 237 duration [14]. In the other method, the single most represen- 238 tative speed-time trace is selected among a large number of 239 


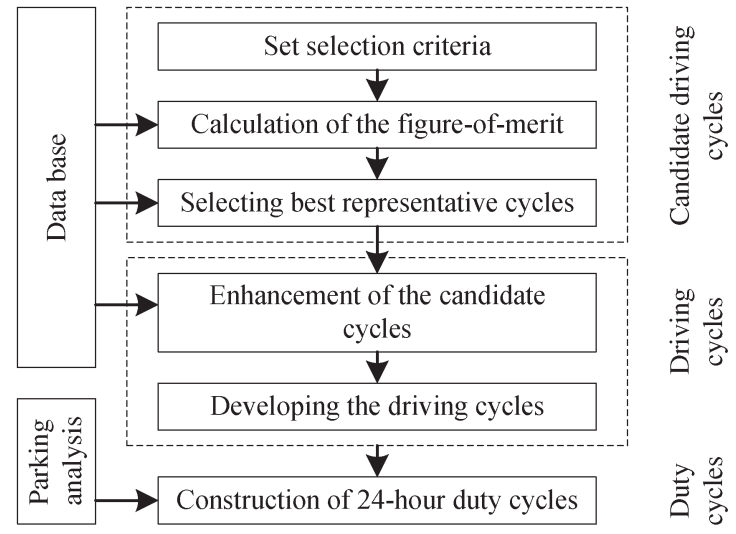

Fig. 2. Methodology of developing a 24-h duty cycle.

240 speed-time traces recorded from real-world driving. A set of 241 characterizing parameters describing the driving cycle is used 242 to select this representative cycle, and modifications are made 243 to the selected cycle to meet certain constraints of import244 ance [17].

245 In this paper, emphasis is placed on the energy and power246 demand aspects of a cycle to address the concerns in plug247 in vehicle design and optimization, as mentioned previously. 248 Therefore, the second methodology is used in this study to 249 develop a cycle realistically mirroring the characteristics of 250 urban driving. This study adopts an adequately long-term ap251 proach to data collection from a fleet of instrumented vehicles 252 to reduce the risk of unreal driving behavior resulting from 253 any influence of the onboard instruments that may potentially 254 bias drivers' driving behavior. Over a one-year timescale, the 255 vehicle owners presumably drive on their ordinary travel routes, 256 whereas onboard instruments automatically timestamp the ve257 hicle's location and speed on a secondly basis.

258 In the present study, data from 76 participant vehicles over a 259 one-year period starting from May 2008 to June 2009 collected 260 by the University of Winnipeg are used. The participants have 261 been selected from different income brackets, education levels, 262 and gender and from different areas of the city to create a 263 statistical population best representing the drivers in the area. 264 The fleet of participating vehicles consists of sedans, both full265 and midsize (67\%), and sport utility vehicles and pickup trucks $266(33 \%)$. Recorded raw data are used to create a database for 267 further analysis to identify daily driving cycles and parking 268 times. The sources of error in collection of data for this study 269 can be attributed to GPS loss-of-signal in the beginning of some 270 trips due to lack of connection to the positioning satellites or 271 blockage due to tall buildings in the downtown area or during 272 the days with significant cloud coverage.

273 The database includes trip number, date, time, position, 274 actual speed, and maximum allowable speed (according to the 275 traffic signs at a vehicle's location), on a secondly basis. The 276 recorded driving cycles of participating vehicles are divided 277 into two groups of weekday and weekend cycles. Commercial 278 fleet users were excluded from this study. The methodology 279 used in this study is schematically shown in Fig. 2.

280 It comprises three major steps to find a candidate cycle, 281 enhancing the candidate cycle for best representativeness, and, finally, including results of parking data analysis into the 282 driving cycle to create a 24-h duty cycle.

In this paper, two daily driving cycles, i.e., one representing 284 weekdays and one for weekends, are selected among the avail- 285 able recorded cycles to avoid lower resolution resulting from 286 mixing their different parking and driving patterns. The two 287 candidate driving cycles best match the average weekday and 288 weekend behavior of the fleet in terms of a set of characterizing 289 parameters given in Table I.

290

The objective of analyzing parking data, which constitute 291 the potential charging times for plug-in vehicles, is twofold: 292 1) to optimize the battery size for an individual vehicle based 293 on several realistic charging scenarios and 2) to estimate the 294 hourly distributed load on the electric grid of the municipality 295 for preparation of adequate infrastructure to keep pace with 296 increasing popularity of plug-in vehicles in the future. The 297 former is important from a vehicle manufacturer's perspective, 298 and the latter is crucial for the electric utility to locate high- 299 voltage feeders and redesign required infrastructure to charge 300 vehicles in urban areas.

\section{A. Selecting the Candidate Driving Cycles}

A set of 25 parameters, as listed in Table I, is used to 303 characterize each of the driving cycles in the pool of recorded 304 data. In addition to the parameters describing kinematics of a 305 cycle, average power demand and average breaking power are 306 also used. This is to extend the set of performance measures 307 for driving cycles in line with the objectives of this study for 308 plug-in vehicle design.

Characteristic parameters of each individual cycle are mea- 310 sured against their corresponding average values, and a figure 311 of merit is calculated and assigned to each individual cycle as 312 follows:

$$
\sigma=\left(\sum_{i=1}^{N}\left(\frac{x_{i}-\overline{x_{i}}}{\overline{x_{i}}}\right)^{2} / N\right)^{1 / 2}
$$

where $\sigma$ is the figure of merit, and $N$ is the number of char- 314 acterizing parameters, which is 25 in this study. Table I shows 315 the list of the characterizing parameters $x_{i}$, and their average 316 values $\bar{x}_{i}$ for both the weekday and weekend cycles are then 317 calculated, as given in Table I.

The parameters in Table I were selected to ensure that the 319 resulting cycle could be used to optimize a large array of 320 drive-train topologies from conventional gas powered to purely 321 electric and with all possible topologies in between when using 322 combinations of propulsion systems.

The cycles that have the closest set of characterizing pa- 324 rameters to the average values in the weekday and weekend 325 groups are selected and will be referred to as the candidate 326 cycles hereinafter. The power and energy demand needed to 327 meet the instantaneous speed of vehicle are calculated based 328 on a longitudinal model for the dynamics of the vehicle, as 329 given in (2) and (3) [31]. The power demand is calculated by 330 integrating net forces acting in the direction of motion over 331 time. The acting forces are the aerodynamic drag $F_{D}$, the time 332 derivative of momentum in the moving direction $m d v / d t$, the 333 
TABLE II

VAlues of PARAmeters USED IN POWER AND ENERGy CALCUlation

\begin{tabular}{ccccccr}
\hline \hline Parameter & $\rho$ & $A_{f}$ & $C_{D}$ & $V_{W}$ & $m$ & $\theta$ \\
Value & $1.2 \mathrm{~kg} / \mathrm{m}^{3}$ & $2.5 \mathrm{~m}^{2}$ & 0.3 & $0 \mathrm{~m} / \mathrm{s}$ & $1550 \mathrm{~kg}$ & 0 \\
\hline \hline
\end{tabular}

334 rolling friction $F_{F}$, and the road grade force $F_{G}$ [32], [33]. The 335 governing equations are given as follows:

$$
\begin{aligned}
& P=\left(m \frac{d v}{d t}+F_{D}+F_{F}+F_{G}\right) v \\
& E=\int_{0}^{t}\left(m \frac{d v}{d t}+F_{D}+F_{F}+F_{G}\right) \cdot v \cdot d t
\end{aligned}
$$

336 where

$$
\begin{aligned}
& F_{D}=\rho A_{f} C_{D}\left(v+v_{w}\right)^{2} / 2 \\
& F_{F}=(1+v / 100) \cdot m g \cdot \cos \theta / 100 \\
& F_{G}=m g \sin \theta
\end{aligned}
$$

$337 \rho$ is the air density, $A_{f}$ is the vehicle frontal area, $C_{D}$ is 338 the aerodynamic drag coefficient, $V_{W}$ is the head-wind speed, $339 \mathrm{~m}$ is the mass of the vehicle, $g$ is the gravitational constant $340\left(9.8 \mathrm{~m} / \mathrm{s}^{2}\right)$, and $\theta$ is the grade angle of the road. In this paper, 341 typical values for a midsize sedan, as given in Table II, are used 342 in the energy and power calculations.

343 This assumption is in line with the dominance of sedans in 344 urban transportation fleet, which is also mirrored in the fleet of 345 participant vehicles in the data-collection phase. Positive values 346 of power demand indicate the power to be provided by the 347 propulsion system at the wheels. The total daily energy demand 348 is calculated by integrating the positive values of the power 349 demand over time divided by the number of data-collection 350 days. Negative values of power demand represent the power 351 to be dissipated as heat by conventional breaking systems or 352 partially recovered in regenerative breaking systems.

\section{B. Enhancement of the Candidate Driving Cycles}

354 It is clear that the two candidate driving cycles do not 355 necessarily match all the average values uniformly well, al356 though they have the best figures of merit for single daily 357 cycles in the database. In this paper, for instance, figures of 358 merit for weekday and weekend candidate cycles are 0.197 359 and 0.295 , respectively. To enhance the quality of the candidate 360 cycles, further processing is done with a view to improve their 361 figure of merit using microtrips of other cycles available in 362 the database. The candidate cycles are then enhanced based 363 on a methodology shown in Fig. 3. A maximum 5\% deviation 364 from average daily energy demand for final driving cycles is 365 allowed in the construction of the enhanced candidate cycles. 366 The average values for the enhanced candidate cycles are also 367 given in Table I.

368 The recorded speed-time traces from the database are split 369 into "microtrips." A microtrip is defined as a snippet of the 370 speed-time trace that begins and ends at idle states: zero speed. 371 The microtrips are then classified according to their traffic

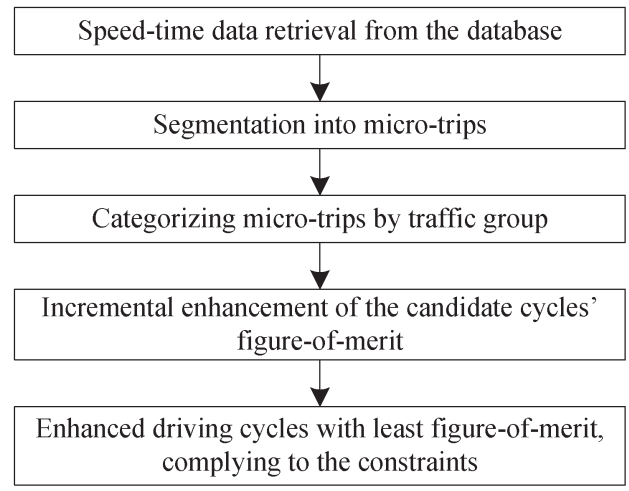

Fig. 3. Methodology of enhancing the candidate cycles.

TABLE III

MICROTRIP CLUSTERING CRITERIA

\begin{tabular}{lll}
\hline \hline Traffic category & Average speed & \multicolumn{1}{c}{ Acceleration } \\
\hline Congested & Low: $[05] \mathrm{km} / \mathrm{h}$ & Mild: $[-0.1,0.1] \mathrm{m} / \mathrm{s}^{2}$ \\
Urban & Moderate: $[5,40] \mathrm{km} / \mathrm{h}$ & Harsh: $[-3.0,3.0] \mathrm{m} / \mathrm{s}^{2}$ \\
Highway & High: $[40100] \mathrm{km} / \mathrm{h}$ & Moderate: $[-1.0,1.0] \mathrm{m} / \mathrm{s}^{2}$ \\
\hline \hline
\end{tabular}

groups characterized by average speed and acceleration, as 372 given in Table III. Here, each microtrip of the candidate cycle is 373 iteratively exchanged with microtrips of the same traffic group 374 until the best figure of merit $\sigma$ is obtained.

375

Classification of microtrips is an important step in the 376 cycle-enhancement method that describes their physical char- 377 acteristics in terms of driving patterns and traffic conditions. 378 Congested traffic such as stop-and-go patterns is characterized 379 by low average speed and mild acceleration (e.g., driving pat- 380 tern in main commuting streets during rush hours). Urban traffic 381 is designated by its moderate average speed and wider range 382 of acceleration typically governed by stop signs and traffic 383 lights in normal urban driving. Finally, the distinct feature of 384 highway traffic is high average speed and moderate acceleration 385 rates. The variations in the speed and acceleration can change 386 the power demand accordingly, and hence, the time percent- 387 ages spent in various speed and acceleration ranges provide 388 important information about power demand in different traffic 389 categories defined in Table III. $\quad 390$

Speed-acceleration frequency distribution (SAFD) plots pro- 391 vide the needed information about the time proportions of 392 individual driving modes [34]. The use of microtrips of the 393 same traffic group serves to maintain the matching of the SAFD 394 of the two candidate cycles to that of the SAFD of all recorded 395 cycles.

An alternative approach to developing a driving cycle is to 397 use random selection methods to select the appropriate number 398 of classified microtrips required to construct a representative 399 cycle matching well with the average characterizing parame- 400 ters with the lowest figure of merit. Random combination of 401 microtrips has been used by other researchers as a means to 402 construct representative driving cycles [35]. Another approach 403 to improve the current methodology would be to give a weight- 404 ing factor to the terms in the definition of the figure of merit [see 405 (1)] to adjust sensitivity of the final result to bias characterizing 406 parameters in accordance with their importance in the final 407 

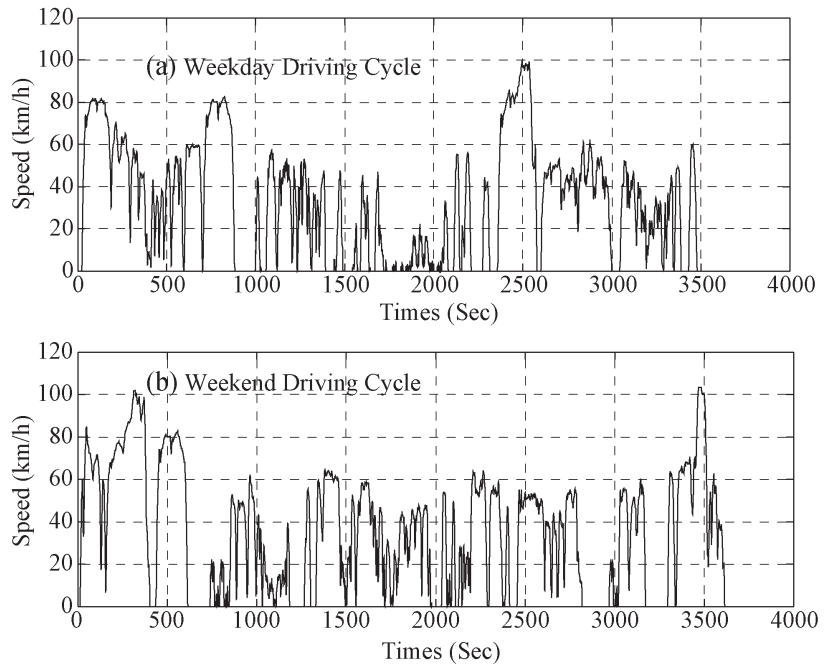

Fig. 4. Enhanced candidate driving cycles. (Top) Weekday. (Bottom) Weekend.

408 duty cycle. In this paper, all weighting factors are considered 409 equal to 1 . Evidently, energy needed and power demand for 410 traveling the same distance in different traffic modes are not 411 equal. It is also well understood that the aggressiveness of the 412 driver in accelerating and decelerating the vehicle increases 413 power consumption. However, it should be noted that replacing 414 microtrips of the candidate cycle with microtrips of the same 415 traffic mode, but potentially from different driving styles, is 416 not misleading from an energy perspective. This is due to the 417 fact that all parameters defining aggressiveness, energy level, 418 and power consumption are already included in the 25 char419 acterizing parameters used in this study, and the replacements 420 increasing the figure of merit to larger values are not con421 sidered. Implementing alternative enhancement methodologies 422 mentioned earlier and their performance assessment are left for 423 further work. Fig. 4 shows the enhanced weekday and weekend 424 candidate driving cycles. The metric units are used throughout 425 the study.

426 The speed in the driving cycles shown in Fig. 4 is given 427 in kilometers per hour; however, for more convenience, both 428 English and metric versions of the driving cycles in digital 429 format are made available to the public [36].

430 Durations of the weekday and weekend cycles are 3484 and $4313616 \mathrm{~s}$, respectively. The maximum velocity is higher in the 432 weekend cycle, i.e., $114 \mathrm{~km} / \mathrm{h}$, whereas in the weekday cycle, 433 the maximum velocity is $89.6 \mathrm{~km} / \mathrm{h}$. The enhancement process 434 does not necessarily finish by yielding a figure of merit equal to 435 zero, but a considerable improvement can be expected as, in this 436 study, the initial values were improved by approximately $40 \%$. 437 The figure of merit for the enhanced weekday and weekend 438 driving cycles are 0.15 and 0.2 , respectively. Fig. 5 shows 439 the SAFD plot for weekday and weekend enhanced candidate 440 driving cycles.

441 The two patterns are different in nature. Stop-and-go events 442 characterized by larger acceleration or deceleration rates at low 443 speeds are more probable in the weekday pattern. However, 444 high-speed events are more probable in the weekend pattern. 445 The driving pattern on the weekend is slightly more aggressive
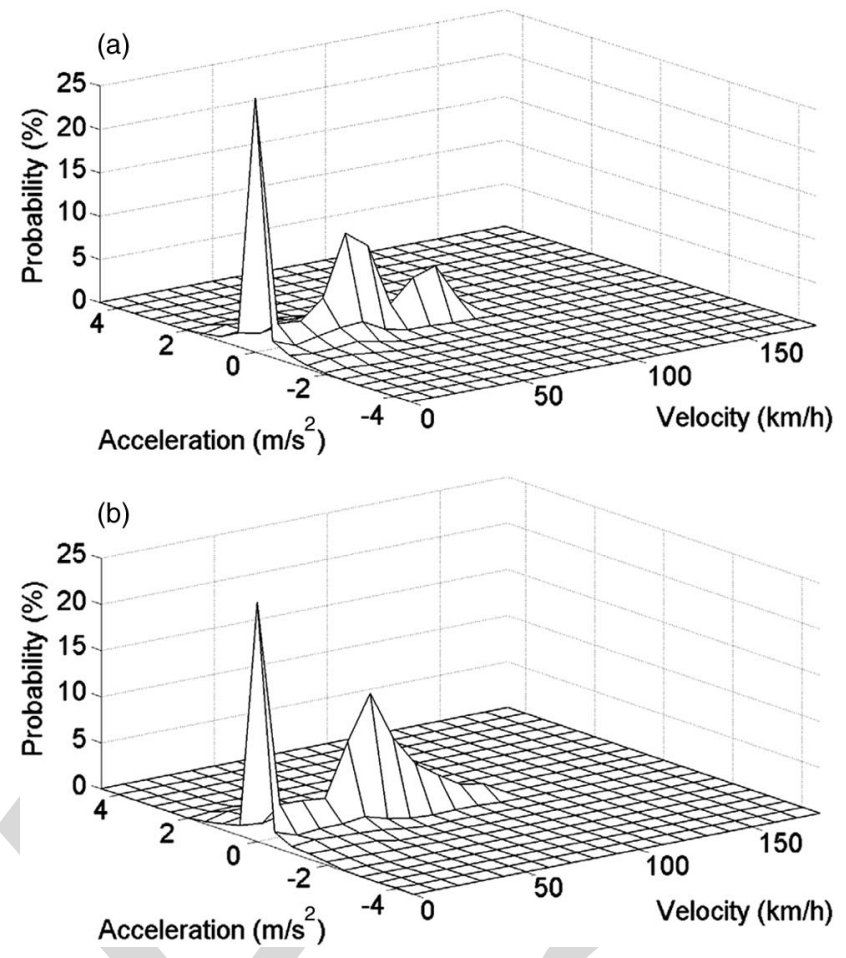

Fig. 5. SAFD plots for the enhanced candidate driving cycles. (a) Weekday. (b) Weekend.
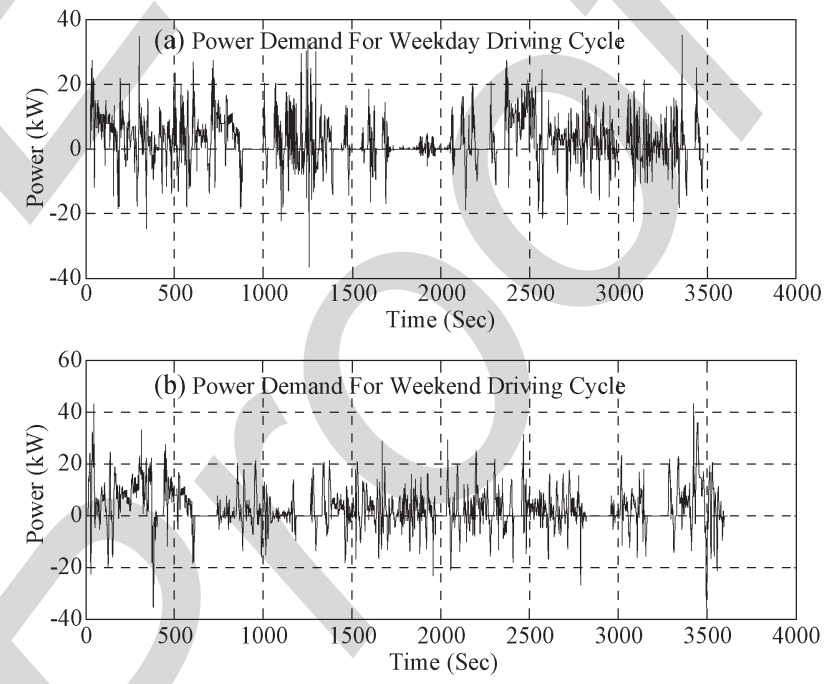

Fig. 6. Power-time traces for the enhanced candidate driving cycles. (a) Weekday. (b) Weekend.

due to higher acceleration and deceleration rates, which results 446 in higher power demand for weekend driving patterns.

The power-time profiles corresponding to the two driving 448 cycles are also presented in Fig. 6. To obtain these profiles, a 449 vehicle with the specifications listed in Table II is considered 450 to have driven the two cycles. Evidently, variations and abrupt 451 changes in power demand are more considerable in the stop- 452 and-go driving mode that is dominant in congested and urban 453 traffics in the weekday driving cycle. This mode of driving 454 considerably contributes to air pollution in downtown areas of 455 large metropolitan areas, and it is particularly important to be 456 covered by electric propulsion in the case of HEVs. 


\section{PARKING ANALYSIS}

459 Vehicle parking at home, the workplace, commercial lo460 cations, and on the street constitutes a critically important 461 element of a modern duty cycle that can address a multitude 462 of drive-train topologies, storage technologies, and controllers. 463 Developing models to analyze the parking behavior in an urban 464 area for city planning may require detailed information on the 465 parameters affecting parking behavior during the day, such as 466 travel demands, district-based knowledge on cost of parking, 467 nature of activities in the area of interest, and supply and 468 demand on an hourly basis. However, from the charging per469 spective only, relatively long parking times provide potential 470 charging times to increase the SOC of an onboard energy 471 storage device. A typical duration for a full charge under normal 472 charging conditions ( $110 \mathrm{~V}$ and $15 \mathrm{~A}$ ) for current competitive 473 battery technologies used in electric vehicles, namely, lithium 474 ion and nickel metal hydride, is approximately $6 \mathrm{~h}$; the mini475 mum duration for partial charging is presently not known with 476 a high confidence level. Therefore, it is expected that most 477 plug-in electric vehicles will be charged mainly overnight. If 478 a relatively long parking time (e.g., more than $3 \mathrm{~h}$ ) during the 479 day is considered as a realistic scenario for a reasonable share 480 of the urban fleet, it is possible to downsize the battery storage 481 capacity and reduce the capital cost of a plug-in vehicle or, 482 in the case of a PHEV with a fixed size of battery storage, 483 drive more miles on electricity to improve cost effectiveness. 484 In addition, fast charging schemes using level $2(120 \mathrm{~V}$ and $48530 \mathrm{~A})$ and direct dc chargers will facilitate full charging in 486 shorter charging durations, i.e., as low as $20 \mathrm{~min}$, depending 487 on the battery technology and dc charging infrastructure.

488 It is also important to highlight that identification of parking 489 locations for charging opportunities is critical; this aspect has 490 been of strong interest to utilities, as they need to address both 491 power and energy demand for electric mobility, with power 492 having a large impact on grid infrastructure costs. Charging 493 at residential areas, parking lots at work places, and large 494 shopping malls is of interest; however, parking in streets or 495 less-frequently open areas is not emphasized due to the large 496 infrastructure cost required to achieve this type of opportunity 497 charging. In the development of a duty cycle in this work, 498 parking events are described for all categories over a 24-h 499 period to provide data for any combination of future charging 500 scenarios. Inherent in this study is the assumption that plug501 in vehicle owners will not be significantly modifying their 502 parking behaviors, although they may change their parking stall 503 to access a plug.

504 An extensive literature survey reveals that a few studies 505 have focused on the potential charging aspect of parking times 506 as a part of daily activities of vehicles; none seem to have 507 incorporated this into a duty cycle. This aspect is of critical 508 importance to address energy drivers in transportation and 509 allow the displacement of fossil fuels with new renewable 510 energy generation. The analysis conducted by the Argonne 511 National Laboratory (ANL) shows that, in the United States, $51266 \%$ of the vehicles driven to work are parked more than $5133 \mathrm{~h}$ before noon, potentially allowing a second charge before 514 the electric utility peak demand begins [37]. It was also ob515 served that vehicles were parked for a short time for shop-
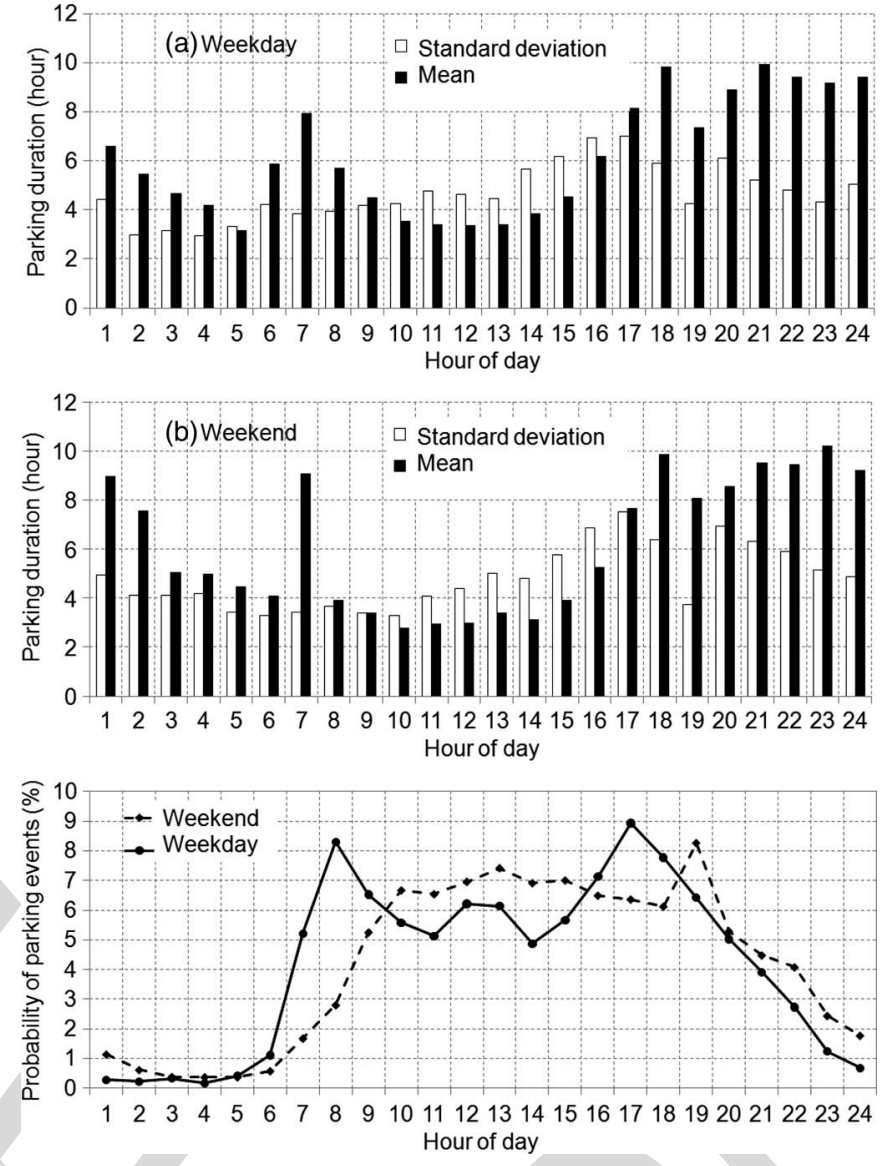

Fig. 7. Mean and standard deviation of parking duration by hour of day. (a) Weekdays. (b) Weekends. (c) Probability of parking events by hour of day for both weekdays and weekends.

ping purposes, and parking was often during peak electric 516 demand [36].

In this paper, GPS-based data loggers provide information 518 on location, type of parking, and duration of parking events. 519 The type of parking can be determined from the driver's 520 behavior, where it is relatively simple to determine where 521 the person lives and works; commercial parking locations 522 are found by digitizing commercial parking lots in Winnipeg. 523 Street parking is deduced from a car staying on the street. Note 524 that studies dedicated to record the instantaneous speed of the 525 vehicle for certification purposes, such as that leading to the 526 FTP72 standard cycle and its enhanced cycles, do not provide 527 information on parking times. Here, probable parking times 528 and average duration for each parking event, as well as the 529 standard deviation of the data points, are included to adequately 530 reflect a daily driving and parking profile for electric vehicle 531 design of drive-train topologies.

Parking times of less than half an hour are arbitrarily clas- 533 sified in our study as short; the distribution of such parking 534 events shows that, in early mornings and afternoons, this type 535 of parking is the most likely. It is important to note that stop 536 times of less than $2 \mathrm{~min}$, happening at stop signs or traffic 537 lights, are excluded from short parking. The results of short 538 parking times are not presented here as it is assumed that, in 539 the real world, these occasional parking events are not favored 540 by drivers for charging. However, a cumulative parking time 541 
542 representing short parking times per day will be included in the 543 final duty cycles. The results of studying parking periods that 544 exceed 30 min are shown in Fig. 7.

545 Fig. 7(a) and (b) shows mean values and standard deviations 546 of parking durations by hour of day for weekdays and week547 ends, respectively.

548 Two distinct patterns in daily parking behavior can be recog549 nized, namely, a mean value less than or equal to the standard 550 deviation and a mean value larger than the standard deviation. 551 Long parking duration with a mean value larger than the stan552 dard deviation happens between 8 P.M. and 6 A.M., showing 553 that the majority of drivers tend to park their vehicles for a long 554 time. However, when the standard deviation is greater than the 555 mean value of parking duration, the data show an increase in 556 daily trips, happening between 10 A.M. and 4 P.M., and the 557 average of the mean values is about $4 \mathrm{~h}$. Peaks in probability 558 of parking events in terms of hour of day, as shown in Fig. 7(c), 559 reflect the difference in nature of activities between weekdays 560 and weekends. While there is only one peak in the weekend 561 curve happening at 7 P.M., there are three peaks occurring at 562 7A.M., 12 P.M., and 5 P.M. during weekdays.

563 On weekdays, $67 \%$ of the vehicles park for more than $3 \mathrm{~h}$ be564 tween 6 A.M. and 9 A.M., whereas between 9 A.M. and 12 P.M., 565 only $33 \%$ of the vehicles park for more than $3 \mathrm{~h}$. This can 566 be justified as many commuters drive to work and park their 567 car during working hours at their working place early in the 568 morning. However, after 9 A.M., vehicles moving in the streets 569 tend to park for a limited duration, i.e., typically less than $3 \mathrm{~h}$, 570 which is necessary for activities such as shopping. The analysis 571 shows that, while overnight charging is the first choice for 572 charging the battery, second charging is most likely to happen 573 in the morning around 9 A.M. or in the afternoon around 5 P.M. 574 Taking the higher electricity price in peak hours in many 575 jurisdictions, a more realistic scenario for charging would be 576 overnight followed by early mornings. However, the distribu577 tion of charging load on the electric grid overnight or during the 578 day would be different, which indicates a need to redesign the 579 location of feeders in the city. This aspect of charging electric 580 cars is beyond the scope of this study and will be published in 581 separate articles of various charging opportunities and power 582 levels.

\section{Assembly of the Driving CyCles}

584 Analysis was performed on the raw GPS data in conjunction 585 with the digitized maps of the roads and commercial parking 586 locations to characterize the driving and parking behavior of 587 the vehicles under the one-year span of the survey in the 588 city of Winnipeg. Using the method shown in Fig. 2, two 589 24-h vehicle usage profiles representing a daily duty cycle 590 were developed for both weekdays and weekends. The parking 591 patterns obtained from the analysis of parking times, as well as 592 cumulative short parking events, are included in the daily duty 593 cycles for weekdays and weekends. In creating this, the average 594 distance traveled in driving events is considered to separate 595 the final driving cycle into parts, and then, parking events are 596 inserted in between in the most probable way. The resulting 597 cycles are shown in Fig. 8 and are meant to represent the
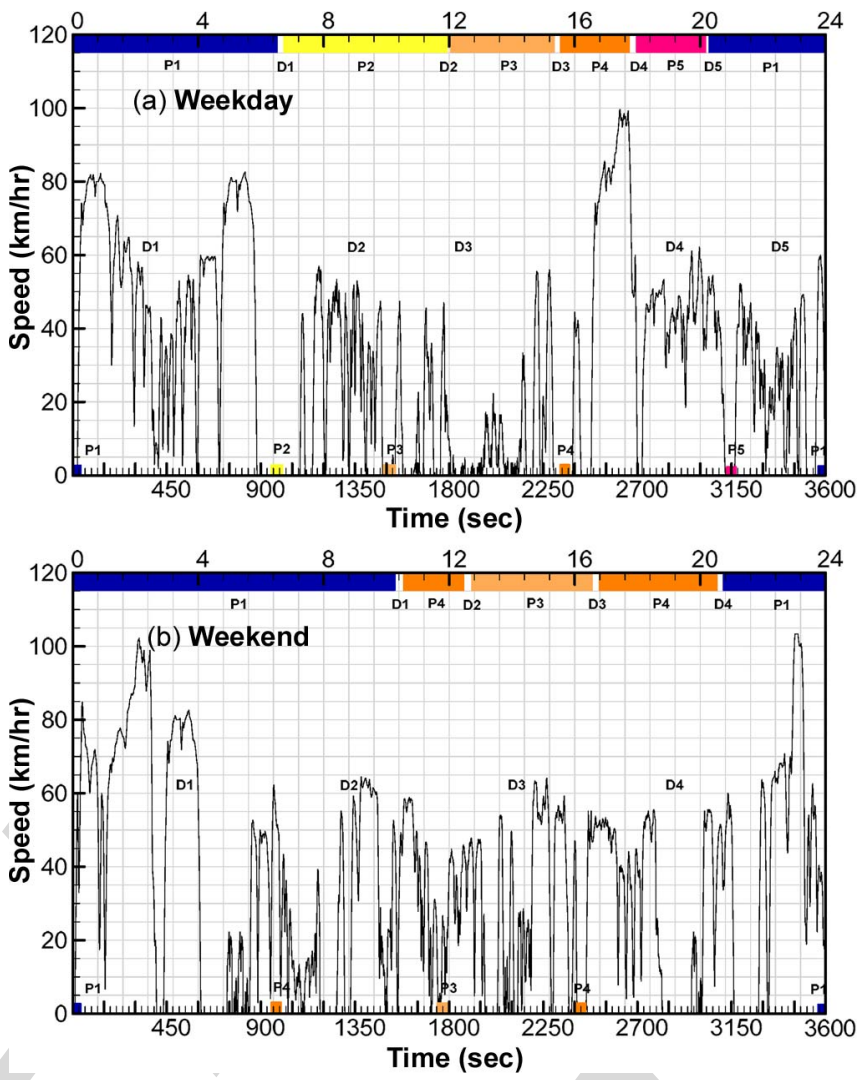

Fig. 8. Resulting 24-h duty cycles. (a) Weekdays. (b) Weekends.

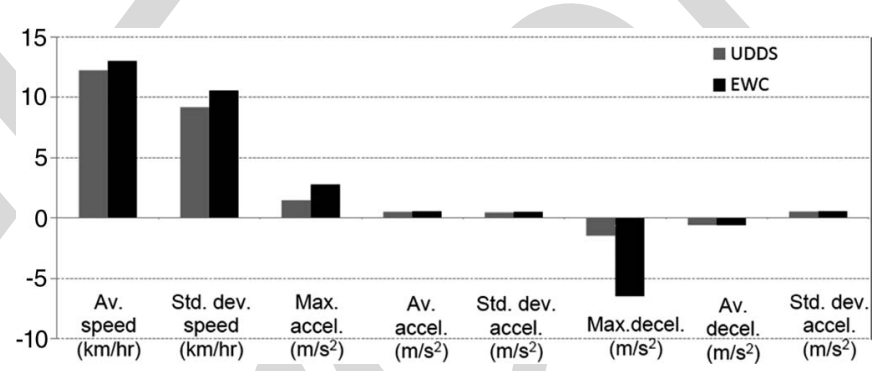

Fig. 9. Comparison between the standard cycle UDDS and the enhanced weekday cycle (EWC).

44 million data points into a condensed duty cycle for studies 598 pertaining to plug-in hybrids, including optimization of power 599 trains [29]. In Fig. 8, D stands for driving, P stands for parking 600 periods, P1 stands for home, P2 stands for work, P3 stands 601 for commercial, P4 stands for short stops, and P5 stands for 602 street parking. The driving cycles are on a 1-h basis, and the 603 duty cycles, with long parking times included, are on a 24-h 604 basis and are both combined into the same figure. The parking 605 durations on a 24-h scale designated by color codes are also 606 shown on a 1-h driving scale for the sake of clarity. The parking 607 events that potentially can be used for charging are P1, P2, or P3 608 when the vehicle is most probably parked in a parking spot with 609 access to level-1 or level-2 charging. The parking events that 610 happen on the street or short parking durations are considered 611 not suitable for charging.

612

Some characteristics of the enhanced driving cycle are com- 613 pared with those of the standard cycle UDDS, and the results 614 are presented in Fig. 9. The comparison indicates that more 615 aggressive characteristics are associated with the real-world 616 
617 cycle, whereas, on average, the two cycles may be considered 618 interchangeable.

619 Evidently, the vehicles with the opportunity to charge limited 620 to overnight have more time to be fully charged under slow 621 charging mode on the weekends. This is particularly important 622 for PEVs with larger battery storage capacity. Deriving a grid 623 load based on this driving cycle has merit but requires the 624 understanding of its limitations for utilities; however, it is 625 beyond the scope of this work.

626 The data files of the duty cycles and the collected raw data 627 are available to the public on the World Wide Web through a 628 unique Digital Object Identifier number [36]. Forty-four mil629 lion speed-time data points, stamped with date and time, and 630 collected over the course of one year are made available. The 631 latitude and longitude of the position of the vehicles recorded 632 on a secondly basis are masked by mapping the starting point of 633 every trip to $(0,0)$ to respect the confidentiality agreement with 634 the participants in the data-collection phase. However, personal 635 information about the participants is used to label the parking 636 locations as home or work. Labeled parking locations, as well 637 as the duration of parking events needed for further analyses 638 with different charging scenarios in the case of plug-in electric 639 vehicles, are included in the data files. The parking events that 640 are less than $30 \mathrm{~min}$ in duration are labeled short stops. Parking 641 events happening along the street are not potentially suitable 642 for charging. The locations of the parking events longer than $64330 \mathrm{~min}$ in duration are marked home, work, shop, and street. The 644 effectiveness of the methodology presented in this study, even 645 with far fewer data points (about 1 million data points, which 646 is equal to about $2 \%$ of the data points used in this study), 647 for simulation-based optimization of a PHEV was shown in [29].

\section{CONCLUSION}

649 A new approach to the development of a duty cycle that ad650 dresses the requirements associated with the design of electric 651 vehicles-e.g., HEV, PHEV, BEV, and extended-range vehi652 cles, has been proposed and implemented on a $24-\mathrm{h}$ timescale. 653 It provides a complete data set for optimization of battery 654 size for on-road vehicles in a typical North American urban 655 setting. For example, power and energy demand in the daily 656 operation of a sedan is directly related to the rate of acceleration 657 and deceleration and time spent in different traffic modes; 658 charging scenarios depend on parking times and duration. The 659 driving behavior of a fleet of 76 participants in a one-year 660 voluntary data-collection program in the city of Winnipeg is 661 analyzed to develop a driving cycle and is composed of two 662 24-h duty cycles for weekdays and weekends. This cycle pro663 vides information about the time and duration of driving in 664 different traffic categories, as well as information on parking 665 times when the vehicle is not in use. Further vehicle simu666 lation tools can use the daily duty cycles developed to op667 timally design propulsion systems, drive-train configurations, 668 and storage components for PEV technologies under real-world 669 driving conditions. Furthermore, this information can be used 670 to analyze the impact of daytime charging by a fleet of plug671 in electric vehicles on the electric utility grid that may create a 672 peak demand during the day to be met by the local utility grid.
The target use of the developed cycle is to provide a duty cycle 673 that can be used to optimally address energy drivers simultane- 674 ously facing transportation by displacing fossil fuels with new 675 renewable energy generations with the direct consequences of 676 increasing the renewable energy ratio of various jurisdictions. 677

To achieve this goal, 25 parameters characterizing a driving 678 cycle for further PEV simulations are recognized, and two 679 candidate daily cycles having the closest match to the average 680 of the parameters are selected. The candidate cycles are then 681 incrementally enhanced by replacing their microtrips with those 682 extracted from the same traffic group, minimizing a figure of 683 merit defined based on the characterizing parameters. Finally, 684 the processed parking data are included to complete two 24-h 685 duty cycles. The final result is therefore reflecting more accu- 686 rately a realistic driving pattern than driving cycles resulting 687 from methodologies that patch snippets of driving data from 688 different drivers or occasions to make a driving cycle. Although 689 the data collected represent driving behavior in the city of 690 Winnipeg, MB, Canada, the methodology presented here can 691 be extended to any other urban area of interest.

692

There exist a few directions to continue the research on or 693 using the collected data. Other methods for development of a 694 driving cycle may include a stochastic approach for selecting 695 and patching snippets of speed-time traces using a probability 696 matrix [14], [21], which might be enhanced to incorporate 697 power and energy requirements of the vehicle. Future work 698 may also concentrate on one specific driving pattern, for in- 699 stance, a commuter, to develop a dedicated driving cycle best 700 mirroring that particular driving pattern. The driving cycle may 701 also be used for a wide range of applications, such as energy 702 assessment of the vehicles in daily use in urban transportation, 703 analysis of charging scenarios in PHEVs and PEVs, vehicle-to- 704 grid analysis, and statistical assessment of driving cycle vari- 705 ability on hybrid drive-train design. Finding other applications, 706 particularly from a social driving behavior perspective, can also 707 be viewed as an important extension of the work.

708

\section{ACKNOWLEDGMENT}

709

The authors would like to thank Prof. D. Blair and R. Smith 710 from the Department of Geography, University of Winnipeg, for 711 providing the collected travel data. Ongoing discussions with 712 Emerging Energy Systems at Manitoba Hydro, in particular 713 with T. Molinski, are acknowledged. Special thanks to Presen- 714 tech Inc. for integrating the parking study with their proprietary 715 firmware.

\section{REFERENCES}

[1] E. Ungar and K. Fell, "Plug in, turn on, and load up," IEEE Power Energy 718 Mag., vol. 8, no. 3, pp. 30-35, May/Jun. 2010.

[2] J. Tollefson, "Charging up the future," Nature, vol. 456, no. 7221, 720 pp. 436-440, Nov. $27,2008$.

[3] A. A. Frank, "Plug-in hybrid vehicles for a sustainable future," Amer. Sci., 722 vol. 95 , no. 2, pp. 158-165, Mar./Apr. 2007.

[4] T. H. Bradley and A. A. Frank, "Design, demonstrations and sustainability 724 impact assessments for plug-in hybrid electric vehicles," Renew. Sustain. 725 Energy Rev., vol. 13, no. 1, pp. 115-128, Jan. 2009.

[5] C. N. Shiau, C. Samaras, R. Hauffe, and J. J. Michalek, "Impact of battery 727 weight and charging patterns on the economic and environmental benefits 728 of plug-in hybrid vehicles," Energy Policy, vol. 37, no. 7, pp. 2653-2663, 729 Jul. 2009. 
[6] A. F. Burke, "Batteries and ultra capacitors for electric, hybrid and fuel cell vehicles," Proc. IEEE, vol. 95, no. 4, pp. 806-820, Apr. 2007.

7] J. J. Michalek, P. Y. Papalambros, and S. J. Skerlos, "A study of fuel efficiency and emission policy impact on optimal vehicle design decisions," ASME J. Mech. Des., vol. 126, no. 6, pp. 1062-1070, Nov. 2004

8] M. Rahimi and A. Emadi, "An analytical investigation of DC/DC power electronic converters with constant power loads in vehicular power systems," IEEE Trans. Veh. Technol., vol. 58, no. 6, pp. 2689-2702, Jul. 2009.

[9] A. Emadi, M. Ehsani, and J. M. Miller, Vehicular Electric Power Systems: Land, Sea, Air, and Space Vehicles. New York: Marcel Dekker, Dec. 2003

0] M. Ehsani and A. Emadi, "Multiconverter power systems and their applications," J. Elect. Power Compon. Syst., vol. 29, no. 10, pp. 949-963, Oct. 2001.

1] A. Emadi, A. Khaligh, C. H. Rivetta, and G. A. Williamson, "Constant power loads and negative impedance instability in automotive systems: Definition, modeling, stability, and control of power electronic converters and motor drives," IEEE Trans. Veh. Technol., vol. 55, no. 4, pp. 1112 1125, Jul. 2006

2] Federal Test Procedure Review Project: Preliminary Tech. Rep. [Online]. Available: http://www.epa.gov/oms/regs/ld-hwy/ftp-rev/ftp-tech.pdf

3] T. C. Austin, F. J. DiGenova, T. R. Carlson, R. W. Joy, K. A. Gianolini, and J. M. Lee, "Characterization of driving patterns and emissions from lightduty vehicles in California," Calif. Air Resources Board, Sacramento, CA, Final Rep. Contract No. A932-185, 1993.

4] J. Lin and D. A. Neimer, "An exploratory analysis comparing a stochastic driving cycle to California's regulatory cycle," Atmos. Environ., vol. 36, no. 38, pp. 5759-5770, Dec. 2002.

5] S. H. Kamble, T. V. Mathew, and G. L. Sharma, "Development of realworld driving cycle: Case study of Pune, India," Transp. Res. Part D, vol. 14, no. 2, pp. 132-140, Mar. 2009.

6] G. Fontaras, P. Pistikopoulos, and Z. Samaras, "Experimental evaluation of hybrid vehicle fuel economy and pollutant emissions over real-world simulation driving cycles," Atmos. Environ., vol. 42, no. 18, pp. 4023 4035, Jun. 2008

7] W. T. Hung, K. M. Tam, C. P. Lee, L. Y. Chan, and C. S. Cheung, "Comparison of driving characteristics in cities of Pearl River Delta, China," Atmos. Environ., vol. 39, no. 4, pp. 615-625, Feb. 2005.

8] G. H. Tzeng and J. J. Chen, "Developing a Taipei motorcycle driving cycle for emissions and fuel economy," Transp. Res. Part D, vol. 3, no. 1, pp. 19-27, Jan. 1998.

9] A. Esteves-Booth, T. Muneer, H. Kirby, J. Kubie, and J. Hunter, "The measurement of vehicular driving cycle within the city of Edinburgh," Transp. Res. Part D, vol. 6, no. 3, pp. 209-220, May 2001.

[20] M. Andre, "Driving cycle development: Characterization of the methods," in Proc. SAE Int. Spring Fuels Lubricants Meet., 1996, pp. 1-13, Technical Paper Series 961112 .

1] J. Lin and D. A. Neimer, "Estimating regional air quality vehicle emission inventories: Constructing robust driving cycles," Transp. Sci., vol. 37, no. 3, pp. 330-346, Aug. 2003.

22] J. Gonder, T. Markel, M. Tornton, and A. Simpson, "Using global positioning system travel data to access real world energy use of plug in hybrid electric vehicles," Transp. Res. Rec., no. 2017, pp. 26-32, Washington, DC, 2007.

3] B. Liaw and M. Dubarry, "From driving cycle analysis to understanding battery performance in real-life electric hybrid vehicle operation," J. Power Sources, vol. 174, no. 1, pp. 76-88, Nov. 2007.

24] A. Fröberg and L. Nielsen, "Efficient drive cycle simulation," IEEE Trans. Veh. Technol., vol. 57, no. 3, pp. 1442-1453, May 2008.

25] Q. Gong, Y. Li, and Z. Peng, "Trip-based optimal power management of plug-in hybrid electric vehicles," IEEE Trans. Veh. Technol., vol. 57, no. 6, pp. 3393-3401, Nov. 2008.

26] J. P. Markey, "Findings from EPA's study of in-use driving patterns," in Proc. 3rd Annu. CRC-APRAC On-Road Veh. Emissions Workshop, San Diego, CA, 1992.

27] E. Milkins and H. Watson, "Comparison of urban driving patterns," presented at the Soc. Automotive Eng. Conf., Warrendale, PA, 1983, Paper 830939.

28] J. E. Morey, T. Limanond, and D. A. Niemeier, "Validity of chase car data used in developing emissions cycles," J. Transp. Stat., vol. 3, no. 2, pp. 15-28, 2000.

29] E. Tara, S. Shahidinejad, S. Filizadeh, and E. Bibeau, "Battery storage sizing in a retrofitted plug-in hybrid electric vehicle," IEEE Trans. Veh. Technol., to be published.
[30] A. A. Pesaran, T. Markel, H. S. Tataria, and D. Howell, "Battery require- 808 ments for plug-in hybrid electric vehicles-Analysis and rationale," in 809 Proc. 23rd Int. EVS, Anaheim, CA, 2007.

[31] J. Y. Wong, Theory of Ground Vehicles. New York: Wiley-Interscience, 811 2001.

[32] T. D. Gillespie, Fundamentals of Vehicle Dynamics. Warrendale, PA: 813 SAE, 1992.

[33] M. Ehsani, Y. Gao, and A. Emadi, Modern Electric, Hybrid Electric, and 815 Fuel Cell Vehicles: Fundamentals, Theory, and Design. Boca Raton, FL: 816 CRC, 2004.

[34] E. Milkins and H. Watson, "Comparison of urban driving patterns, motor 818 vehicle technology: Progress and harmony," in Proc. 2nd Int. Pacific Conf. 819 Autom. Eng., Tokyo, Japan, 1983, pp. 735-746.

[35] T. C. Austin, F. J. DiGenova, T. R. Carlson, R. W. Joy, K. A. Gianolini, 821 and J. M. Lee, Characterization of Driving Patterns and Emissions From 822 Light-Duty Vehicles in California. Sacramento, CA: Sierra Res., 1993. 823 (Prepared for California Air Resources Board).

[36] S. Shahidinejad, E. L. Bibeau, and S. Filizadeh, Winnipeg Driving Cycle: 825 WPG02. DOI:10.5203/ds_bib_1. [Online]. Available: http://mspace.lib. 826 umanitoba.ca/dspace/handle/1993/3997

[37] Argonne Nat. Lab., Transportation Research Development. [Online]. 828 Available: http://www.transportation.anl.gov/pdfs/HV/548.pdf 829

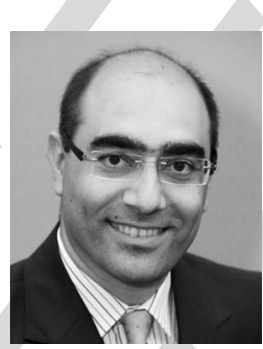

Soheil Shahidinejad received the B.Sc., M.Sc., and 830 $\mathrm{Ph} . \mathrm{D}$. (with honors) degrees from Sharif University 831 of Technology, Tehran, Iran, in 1993, 1996 and 2002, 832 respectively, all in mechanical engineering. $\quad 833$

$\mathrm{He}$ is currently a Research Associate with the 834 Department of Mechanical and Manufacturing 835 Engineering, University of Manitoba, Winnipeg, 836 MB, Canada. His research deals with the study and 837 analysis of energy-efficient systems and renewable 838 energies.

Automotive Engineers. He was a recipient of a one-year scholarship in 84 1999/2000 from the French Government to extend his Ph.D. research with the 842 Institut National Polytechnique de Lorraine, Nancy, France.

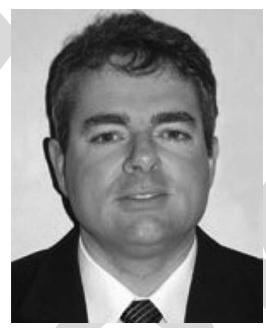

Eric Bibeau received the B.Sc. degree from McGill 844 University, Montreal, QC, Canada, in 1986 and the 845 M.Sc. and Ph.D. degrees from the University of 846 British Columbia, Vancouver, BC, Canada, in 1988847 and 1993, respectively.

848

He currently is an Associate Professor with the 849 University of Manitoba, Winnipeg, MB, Canada, 850 where he holds the National Science and Engineering 851 Research Council of Canada/Manitoba Hydro Chair 852 in Alternative Energy. He specializes in developing 853 power systems using biomass feedstocks, industrial 854 waste heat, and kinetic energy from rivers for distributed energy applications. 855 $\mathrm{He}$ is also involved in developing blade-deicing-mitigation strategies for wind 856 turbines. He is developing expertise in electric vehicles and district energy 857 systems as a means to increase the renewable energy ratio in Canada to 858 simultaneously address climate change and peak oil energy drivers.

Dr. Bibeau is a Registered Professional Engineer in the Province of 860 Manitoba.

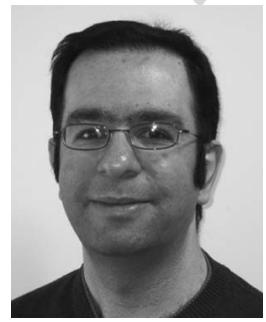

Shaahin Filizadeh (S'02-M'04) received the B.Sc. 862 and M.Sc. degrees in electrical engineering from 863 Sharif University of Technology, Tehran, Iran, in 864 1996 and 1998, respectively, and the Ph.D. degree 865 in electrical engineering from the University of 866 Manitoba, Winnipeg, MB, Canada, in 2004.

$\mathrm{He}$ is currently an Associate Professor with the 868 Department of Electrical and Computer Engineering, 869 University of Manitoba. His areas of interest include 870 power systems electromagnetic transient simulation, 871 nonlinear optimization, and power-electronic appli- 872 cations in power systems and vehicle propulsion.

Dr. Filizadeh is a Registered Professional Engineer in the Province of 874 Manitoba. 


\section{AUTHOR QUERIES}

\section{AUTHOR PLEASE ANSWER ALL QUERIES}

AQ1 = Please provide publication update in Ref. [29].

Notes:

Note that reference [4] and [6] are the same. Therefore, reference [6] was deleted from the list. Citations were renumbered accordingly. Please check.

Note that reference [3] and [7] are the same. Therefore, reference [7] was deleted from the list. Citations were renumbered accordingly. Please check.

\section{END OF ALL QUERIES}




\title{
- Statistical Development of a Duty Cycle for Plug-in Vehicles in a North American Urban Setting Using Fleet Information
}

\author{
Soheil Shahidinejad, Eric Bibeau, and Shaahin Filizadeh, Member, IEEE
}

\begin{abstract}
5 Abstract-Development of a daily duty cycle based on real6 world driving behavior and parking times is a critical requirement 7 in the optimal design of power-train components of a plug-in 8 vehicle. Standard driving cycles cannot completely emulate the 9 real-world power demand of a vehicle and its downtimes in par10 ticular. To address these shortcomings, a large database of one 11 year of measured data collected from a fleet of $\mathbf{7 6}$ cars in the 12 city of Winnipeg, MB, Canada is obtained and is then used to 13 develop a new duty cycle. This paper describes a methodology 14 for statistical analysis of the fleet data, including while a vehicle 15 is parked. Due to the intrinsic differences in vehicle usage profiles 16 during weekdays and weekends, two 24 -h duty cycles with suitable 17 windows of opportunity for charging are developed for weekday 18 and weekend driving patterns. The uniqueness of the proposed 19 statistical methodology and the resulting duty cycles contribute to 20 addressing the present shortcomings of standard driving cycles.
\end{abstract}

21 Index Terms-Battery storage, driving cycle, duty cycle, electric 22 vehicle, plug-in hybrid vehicles, renewable energy.

\section{INTRODUCTION}

24 LECTRIFICATION of transportation for light-duty vehi25 cles is a prominent step toward sustainable transportation 26 [1], [3]. It can also contribute to efficient integration and use 27 of existing and emerging renewable energy resources. Plug-in 28 vehicles (i.e., pure electric or plug-in hybrids) have a strong 29 potential to reduce petroleum consumption by shifting energy 30 demand away from fossil fuels to electrical energy that is 31 domestically produced using renewable sources. A plug-in 32 vehicle allows its battery storage to recharge via connection 33 to a utility grid while the vehicle is parked. Therefore, it 34 covers a wide range of vehicles using electricity as a source of 35 propulsion either partially, such as in a plug-in hybrid electric 36 vehicle (PHEV), or entirely, such as in a battery electric vehicle 37 (BEV). When used in conjunction with a distributed high38 capacity-storage electric utility, it will also help accommodate

Manuscript received April 13, 2010; revised June 21, 2010; accepted July 16, 2010. This work was supported by the AUTO21 Network of Centers of Excellence under Project DF302-DBS. The review of this paper was coordinated by Dr. A. Khaligh.

S. Shahidinejad and E. Bibeau are with the Department of Mechanical and Manufacturing Engineering, University of Manitoba, Winnipeg, MB R3T 5V6, Canada (e-mail: shahidin@cc.umanitoba.ca; bibeauel@cc.umanitoba.ca).

S. Filizadeh is with the Department of Electrical and Computer Engineering, University of Manitoba, Winnipeg, MB R3T 5V6, Canada (e-mail: sfilizad@ee.umanitoba.ca).

Color versions of one or more of the figures in this paper are available online at http://ieeexplore.ieee.org.

Digital Object Identifier 10.1109/TVT.2010.2061243 the variable and unpredictable nature of renewable sources. It 39 is envisioned that by increasing the share of renewable energies 40 for electric power generation and optimizing rechargeable en- 41 ergy storage battery units in plug-in vehicles, major concerns 42 with regard to peak oil, greenhouse gases leading to climate 43 change, energy security, and emissions, can be simultaneously 44 addressed [4]. Due to high cost and large weight per unit energy 45 capacity of current battery cells, the technology pathway for 46 PHEVs to lower the battery size and cost includes providing 47 additional daily charging opportunities during periods when the 48 vehicles are parked and opportunities for charging exist [3]-[7]. 49

Complete assessment of the potential power and energy 50 demand in plug-in vehicles is required to simulate and optimize 51 their energy-storage systems [8]. Optimal sizing of the electric 52 drive-train components, choice of battery chemistry and storage 53 size, development of controllers tuned and optimized to vehicle 54 driving patterns, as well as realistic opportunity charging sce- 55 narios, all require detailed information on the vehicle's usage 56 profile. Obviously, recharging scenarios and grid impacts can be 57 better analyzed with detailed information on parking durations, 58 as well as time and location of parking events in drivers' 59 daily routines. Real-world driving patterns provide insight into 60 speed and acceleration characteristics. However, only stan- 61 dard driving schedules conducted on dynamometers or well- 62 documented tracks have been accepted as a systematic approach 63 to mimic real-life situations. Standard certification driving 64 cycles such as the Urban Dynamometer Driving Schedule 65 (UDDS) or Highway Fuel Economy Test have been convention- 66 ally used in conjunction with controlled chassis dynamometer 67 testing to represent average driving behavior of the drivers in 68 fuel economy and emission certification of the vehicles.

It is important to note that the standard certification cycles are 70 still unable to handle extreme acceleration or deceleration rates 71 that fall beyond capabilities of laboratory equipment and are 72 bound to limited cycle durations, i.e., usually less than $20 \mathrm{~min}, 73$ to keep test costs low [5], [12]. For instance, the FTP72 driving 74 cycle, which dates back to early 1970s, was primarily developed 75 to measure exhaust emissions of typical light-duty vehicle 76 operations in the Los Angeles urban area [13]. To address the 77 shortcomings of the FTP72 cycle in representing more aggres- 78 sive speeds and accelerations, a Unified Cycle was developed 79 in 1992 based on collected data, known as LA92. However, 80 there are still many concerns about the problems inherent in the 81 existing driving cycles, which lead to underestimation of cruise, 82 acceleration, or stop-and-go activities in different brackets of 83 
84 velocities [14]. It is therefore concluded that such cycles cannot 85 completely emulate the real-world daily power demand of a 86 vehicle. More importantly, they do not provide information 87 on parking times as opportunities for charging in the case of 88 emerging plug-in vehicles. In addition, it is important to base 89 duty cycles on larger data sets to reduce statistical errors.

90 An extensive literature survey conducted revealed no refer91 ence to the development of a daily driving cycle, taking into 92 account power and energy demands as major requirements for 93 representing the real-world data. Most studies have focused on 94 developing urban driving cycles using snippets extracted from 95 recorded speed-time traces to estimate vehicular emissions and 96 fuel consumption in different cosmopolitan areas [15], [19]. 97 The objective of these studies is to represent the driving infor98 mation in a collected data set using a single driving cycle. Some 99 other studies discuss the effectiveness of the methodologies 100 used in developing driving cycles to represent the inherent char101 acteristics of driving behavior in the collected data [20], [21].

102 A few recent studies exist that have assessed the performance 103 of hybrid electric vehicles (HEVs) in real-world operation. 104 For example, data collected for a fleet in the St. Louis, MO, 105 metropolitan area were used in the simulation of energy usage 106 in a PHEV, but no single driving pattern was extracted from 107 the collected data [22]. Fuzzy logic pattern recognition tech108 niques have also been used to perform driving and duty cycle 109 analyses on data collected for a fleet of HEVs [23]. Another 110 effort to modify standard cycles for better representing real111 world behavior introduced a driver model in connection with 112 European standard cycles into simulations [24]. A methodology 113 that generates a driving cycle has also been reported based 114 on the assumption of constant acceleration and deceleration 115 rates, along with consideration of the speed limits in different 116 road segments in representative areas [25]. It can therefore be 117 concluded that there does not exist a single widely accepted 118 duty cycle in the literature to appropriately represent typical 119 daily activities of the vehicles and to address the energy and 120 power demands of the PHEVs and BEVs.

121 The study presented in this paper addresses the gap in the 122 literature by developing a statistical methodology and con123 structing the needed duty cycles based on a database of over 12444 million Global Positioning System (GPS) data points 125 recorded over the course of one year in Winnipeg, MB, Canada. 126 Depending on the context, the terms "driving cycle" and 127 "duty cycle" may convey different meanings. In this paper, 128 a "driving cycle" refers to a history of daily driving periods 129 represented by a speed-versus-time curve. A "duty cycle" refers 130 to a profile of daily usage of power by the vehicle, which is 131 typically represented by a 24-h history of driving and parking 132 events. Note that, in the case of an HEV, only a driving cycle 133 is sufficient to calculate vehicle's power demand, whereas in 134 the case of a plug-in vehicle, parking times also become vital 135 as they may be used for charging from the electric grid, and 136 therefore, they should be included in the daily profile. Parking 137 times are also important to utilities servicing jurisdictions with 138 large vehicular loads, as they can be used to predict and control 139 the load on the grid.

140 The objective of the present study is to first develop a 141 new driving cycle most closely mirroring the characteristics of

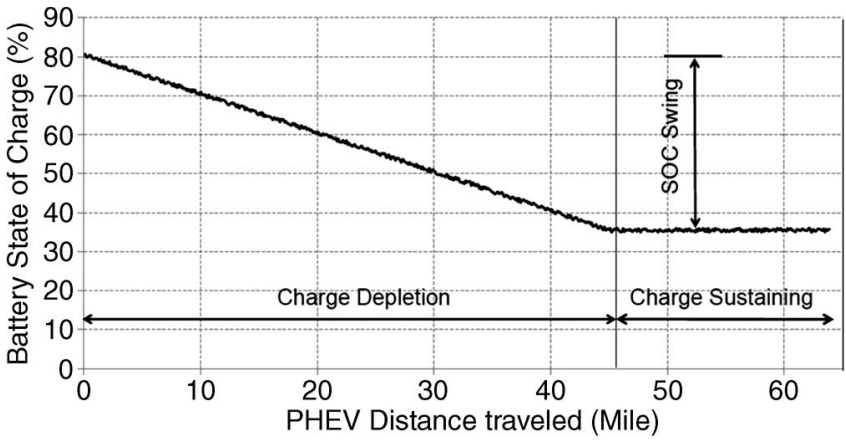

Fig. 1. Typical variation in the SOC of a PHEV battery.

urban driving, including real-world energy and power demands. 142 Once this is achieved, this study aims to incorporate a pattern 143 representing most probable downtimes of the vehicles to charge 144 during their daily usage profile. A comprehensive daily duty 145 cycle is a crucial component for optimal design of plug-in 146 vehicular drive-trains. This study improves the conventional 147 methods of driving cycle generation [26], [28]. The present 148 study also establishes a set of performance measures required to 149 assess a driving cycle suited for electric/plug-in hybrid vehicles. 150 The methodology used in the precedent study for simulation 151 of a plug-in vehicle is enhanced by including 25 parameters 152 to characterize different velocity brackets in a driving cycle 153 [29]. The uniqueness of this study is proposing a methodol- 154 ogy that addresses the particular requirements associated with 155 the design of plug-in vehicles in construction of a 24-h duty 156 cycle.

Following the introduction, in Section II, duty cycle re- 158 quirements to be fulfilled for enhanced simulation and opti- 159 mization of plug-in vehicles are discussed. Data collection, 160 driving cycle generation, and characterization are described 161 in Section III. Parking data analysis for weekdays and week- 162 ends is presented in Section IV. The resulting 24-h duty 163 cycles are given in Section V, and driving characteristics 164 are critically compared with those of the standard cycle for 165 urban driving, i.e., the UDDS. Conclusions are presented 166 in Section VI.

\section{Duty Cycle ReQuirements for Plug-IN Vehicles 168}

The total distance that a plug-in vehicle can electrically 169 drive is an important measure for the vehicle's performance 170 assessment. For instance, PHEVs are usually classified ac- 171 cording to their all-electric range (AER), which is defined as 172 the total miles electrically driven after a full recharge before 173 the engine turns on for the first time [30]. A fully recharged 174 PHEV operates in charge-depleting mode until the battery is 175 depleted to a target state-of-charge (SOC), at which point, the 176 vehicle switches to charge-sustaining mode, using the internal 177 combustion engine to maintain the target SOC [3]. Fig. 1 shows 178 the typical variation in the SOC of the battery in the operating 179 modes of a PHEV.

Obviously, the instantaneous power demand resulting from 181 the driving style of the driver is critical in the definition of the 182 AER. The standard cycle UDDS is usually used to measure 183 
TABLE I

CHARACTERIZING PARAMETERS AND THEIR VALUES

\begin{tabular}{|c|c|c|c|c|c|}
\hline & \multirow{2}{*}{ Parameter } & \multicolumn{2}{|c|}{ Database average } & \multicolumn{2}{|c|}{ Final duty cycles } \\
\hline & & weekday & weekend & Weekday & weekend \\
\hline 1 & Average speed of the entire driving cycle in $\mathrm{km} / \mathrm{h}$ & 31.4 & 35.3 & 32.6 & 35.0 \\
\hline 2 & Average running speed in $\mathrm{km} / \mathrm{h}$ & 39.8 & 43.9 & 40.3 & 43.4 \\
\hline 3 & Total daily distance traveled in $\mathrm{km}$ & 32.2 & 33.9 & 31.6 & 35.2 \\
\hline 4 & Average acceleration of all acceleration phases in $\mathrm{m} / \mathrm{s} 2$ & 0.6 & 0.6 & 0.55 & 0.59 \\
\hline 5 & Average deceleration of all deceleration phases in $\mathrm{m} / \mathrm{s} 2$ & -0.6 & -0.6 & -0.59 & -0.6 \\
\hline 6 & Average number of change in acceleration rate $(+/-)$ in one driving period & 6.5 & 7.2 & 7.83 & 7.9 \\
\hline 7 & Average daily power demand in $\mathrm{kW}$ & 7.9 & 8.3 & 8.92 & 9.3 \\
\hline 8 & Maximum power demand in $\mathrm{kW}$ & 59.3 & 72.2 & 49.45 & 60.0 \\
\hline 9 & Total daily energy demand in $\mathrm{Mj}$ & 17.3 & 17.7 & 16.41 & 18.2 \\
\hline 10 & Average daily breaking power in $\mathrm{kW}$ & -5.3 & -5.4 & -5.47 & -6.0 \\
\hline 11 & Root mean square of acceleration in $\mathrm{m} / \mathrm{s} 2$ & 0.8 & 0.8 & 0.66 & 0.69 \\
\hline 12 & Average length of a driving period in $\mathrm{km}$ & 0.9 & 1.2 & 0.75 & 1.03 \\
\hline 13 & Time percentage of Idling (zero velocity) in \% & 21.8 & 20.7 & 19.1 & 19.31 \\
\hline 14 & Time percentage of acceleration: acceleration $>0.1 \mathrm{~m} / \mathrm{s} 2$ in $\%$ & 31.4 & 31.3 & 36.5 & 35.1 \\
\hline 15 & Time percentage of Cruising (acceleration $[-0.1,0.1] \mathrm{m} / \mathrm{s} 2$, speed $>5 \mathrm{~m} / \mathrm{s}$ ) in $\%$ & 14.9 & 16.7 & 9.8 & 10.5 \\
\hline 16 & Time percentage of deceleration: acceleration $<-0.1 \mathrm{~m} / \mathrm{s} 2$ in $\%$ & 31.2 & 30.9 & 34.4 & 34.9 \\
\hline 17 & Time percentage of creeping (acceleration $[-0.1,0.1] \mathrm{m} / \mathrm{s} 2$, speed $<5 \mathrm{~m} / \mathrm{s}$ ) in $\%$ & 0.6 & 0.5 & 0.32 & 0.14 \\
\hline 18 & $\begin{array}{l}\text { Time percentage of driving at very low speed bracket } \\
{\left[\begin{array}{ll}0 & 20\end{array}\right] \mathrm{km} / \mathrm{h} \text { in } \%}\end{array}$ & 94.5 & 90.6 & 85.3 & 90.5 \\
\hline 19 & Average speed in very low speed bracket $\left[\begin{array}{lll}0 & 20\end{array}\right] \mathrm{km} / \mathrm{h}$ & 6.6 & 10.3 & 7.3 & 6.5 \\
\hline 20 & $\begin{array}{l}\text { Time percentage of driving at low speed bracket } \\
{[2050] \mathrm{km} / \mathrm{h} \text { in } \%}\end{array}$ & 58.9 & 59.6 & 62.0 & 61.0 \\
\hline 21 & Average speed in low speed bracket $\left[\begin{array}{ll}20 & 50\end{array}\right] \mathrm{km} / \mathrm{h}$ & 18.9 & 20.8 & 19.2 & 28.1 \\
\hline 22 & Time percentage of driving at moderate speed bracket $\left[\begin{array}{ll}50 & 80\end{array}\right] \mathrm{km} / \mathrm{h}$ in $\%$ & 36.7 & 36.9 & 37.4 & 36.4 \\
\hline 23 & Average speed in moderate speed bracket $\left[\begin{array}{ll}50 & 80\end{array}\right] \mathrm{km} / \mathrm{h}$ & 32.9 & 30.2 & 34.9 & 29.0 \\
\hline 24 & $\begin{array}{l}\text { Time percentage of driving at high speed bracket } \\
{\left[\begin{array}{ll}80 & 100\end{array}\right] \mathrm{km} / \mathrm{h} \text { in } \%}\end{array}$ & 9.9 & 10.0 & 8.9 & 10.6 \\
\hline 25 & Average speed in high speed bracket $\left[\begin{array}{lll}80 & 100\end{array}\right] \mathrm{km} / \mathrm{h}$ & 16.3 & 15.0 & 16.9 & 15.1 \\
\hline
\end{tabular}

184 the AER for classifying PHEVs. Therefore, a PHEV $x$ expected 185 to travel $x$ miles on battery, in the real world, would perform 186 quite differently, depending on the driving habits of the driver. 187 The mentioned difficulty in providing a realistic performance 188 measure to the end users is also valid in the case of an HEV. 189 The dominant mode of operation in an HEV battery is charge 190 sustaining, and its fuel efficiency is characterized by a higher 191 mile per gallon rate when compared with conventional vehicles. 192 Again, using the standard cycle is misleading as, in reality, fuel 193 consumption would be higher, depending on the aggressiveness 194 of the driver.

195 In this study, real-world driving data are collected over a 196 sufficiently long period to reduce statistical errors. In addition, 197 the characterizing parameters of the driving cycles used to 198 generate the standard driving cycles are extended to cover 199 average driver's daily energy demand and maximum power 200 demand (positive in acceleration and negative in deceleration) 201 for light-duty vehicles (with a dominant share in urban traffic). 202 The information on parking events such as the frequency of 203 occurrence and the ratio of parking to driving times in an 204 average daily driving profile is also included to construct an 205 average 24 -h duty cycle.

206 The characterizing parameters considered in this paper are 207 those defining power requirements at different driving modes, 208 as listed in Table I. Except for its considerably cold winter 209 temperatures, Winnipeg, with a population of 700 000, is a 210 typical North American urban setting where driving culture, 211 population, and traffic behavior are similar to many other cities 212 across the United States and Canada. Although the particular duty cycles developed in this study may be applied to many 213 other similarly populated cities, the methodology described in 214 the next section is general and can be used to develop duty 215 cycles for any other urban area of interest.

\section{Methodology of Development OF A DRIVING CYCLE}

The proposed methodology comprises two stages: 1) de- 219 veloping a driving cycle based on a large set of data points 220 collected and 2) incorporating results of a statistical analysis 221 on daily parking times to construct a 24-h duty cycle. An 222 enhanced statistical approach is developed using 25 parame- 223 ters to characterize the driving cycle by selecting candidates 224 from the database that have the closest match to the average 225 of the parameters. The candidate cycle is then incrementally 226 enhanced by replacing its microtrips with those extracted from 227 the same traffic group to minimize a figure of merit defined 228 based on average values of the characterizing parameters. The 229 processed parking data are then categorized in various groups 230 and included in the daily usage profile.

There are two general methodologies to develop a driving 232 cycle. One is based on creating a pool of trip segments extracted 233 from recorded speed-time traces of vehicles, followed by cat- 234 egorizing them into several driving modes and finally patching 235 snippets selected based on desired selection criteria together 236 to develop a representative driving cycle with a predetermined 237 duration [14]. In the other method, the single most represen- 238 tative speed-time trace is selected among a large number of 239 


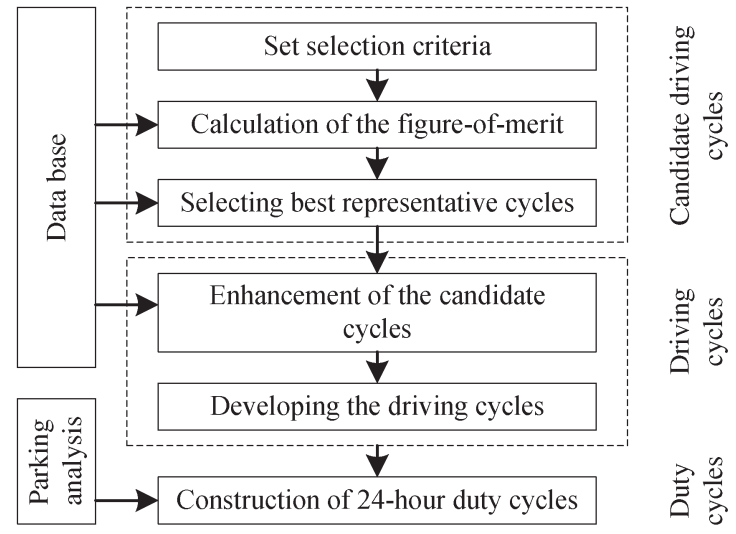

Fig. 2. Methodology of developing a 24-h duty cycle.

240 speed-time traces recorded from real-world driving. A set of 241 characterizing parameters describing the driving cycle is used 242 to select this representative cycle, and modifications are made 243 to the selected cycle to meet certain constraints of import244 ance [17].

245 In this paper, emphasis is placed on the energy and power246 demand aspects of a cycle to address the concerns in plug247 in vehicle design and optimization, as mentioned previously. 248 Therefore, the second methodology is used in this study to 249 develop a cycle realistically mirroring the characteristics of 250 urban driving. This study adopts an adequately long-term ap251 proach to data collection from a fleet of instrumented vehicles 252 to reduce the risk of unreal driving behavior resulting from 253 any influence of the onboard instruments that may potentially 254 bias drivers' driving behavior. Over a one-year timescale, the 255 vehicle owners presumably drive on their ordinary travel routes, 256 whereas onboard instruments automatically timestamp the ve257 hicle's location and speed on a secondly basis.

258 In the present study, data from 76 participant vehicles over a 259 one-year period starting from May 2008 to June 2009 collected 260 by the University of Winnipeg are used. The participants have 261 been selected from different income brackets, education levels, 262 and gender and from different areas of the city to create a 263 statistical population best representing the drivers in the area. 264 The fleet of participating vehicles consists of sedans, both full265 and midsize (67\%), and sport utility vehicles and pickup trucks $266(33 \%)$. Recorded raw data are used to create a database for 267 further analysis to identify daily driving cycles and parking 268 times. The sources of error in collection of data for this study 269 can be attributed to GPS loss-of-signal in the beginning of some 270 trips due to lack of connection to the positioning satellites or 271 blockage due to tall buildings in the downtown area or during 272 the days with significant cloud coverage.

273 The database includes trip number, date, time, position, 274 actual speed, and maximum allowable speed (according to the 275 traffic signs at a vehicle's location), on a secondly basis. The 276 recorded driving cycles of participating vehicles are divided 277 into two groups of weekday and weekend cycles. Commercial 278 fleet users were excluded from this study. The methodology 279 used in this study is schematically shown in Fig. 2.

280 It comprises three major steps to find a candidate cycle, 281 enhancing the candidate cycle for best representativeness, and, finally, including results of parking data analysis into the 282 driving cycle to create a 24-h duty cycle.

In this paper, two daily driving cycles, i.e., one representing 284 weekdays and one for weekends, are selected among the avail- 285 able recorded cycles to avoid lower resolution resulting from 286 mixing their different parking and driving patterns. The two 287 candidate driving cycles best match the average weekday and 288 weekend behavior of the fleet in terms of a set of characterizing 289 parameters given in Table I.

290

The objective of analyzing parking data, which constitute 291 the potential charging times for plug-in vehicles, is twofold: 292 1) to optimize the battery size for an individual vehicle based 293 on several realistic charging scenarios and 2) to estimate the 294 hourly distributed load on the electric grid of the municipality 295 for preparation of adequate infrastructure to keep pace with 296 increasing popularity of plug-in vehicles in the future. The 297 former is important from a vehicle manufacturer's perspective, 298 and the latter is crucial for the electric utility to locate high- 299 voltage feeders and redesign required infrastructure to charge 300 vehicles in urban areas.

\section{A. Selecting the Candidate Driving Cycles}

A set of 25 parameters, as listed in Table I, is used to 303 characterize each of the driving cycles in the pool of recorded 304 data. In addition to the parameters describing kinematics of a 305 cycle, average power demand and average breaking power are 306 also used. This is to extend the set of performance measures 307 for driving cycles in line with the objectives of this study for 308 plug-in vehicle design.

Characteristic parameters of each individual cycle are mea- 310 sured against their corresponding average values, and a figure 311 of merit is calculated and assigned to each individual cycle as 312 follows:

$$
\sigma=\left(\sum_{i=1}^{N}\left(\frac{x_{i}-\overline{x_{i}}}{\overline{x_{i}}}\right)^{2} / N\right)^{1 / 2}
$$

where $\sigma$ is the figure of merit, and $N$ is the number of char- 314 acterizing parameters, which is 25 in this study. Table I shows 315 the list of the characterizing parameters $x_{i}$, and their average 316 values $\bar{x}_{i}$ for both the weekday and weekend cycles are then 317 calculated, as given in Table I.

The parameters in Table I were selected to ensure that the 319 resulting cycle could be used to optimize a large array of 320 drive-train topologies from conventional gas powered to purely 321 electric and with all possible topologies in between when using 322 combinations of propulsion systems.

The cycles that have the closest set of characterizing pa- 324 rameters to the average values in the weekday and weekend 325 groups are selected and will be referred to as the candidate 326 cycles hereinafter. The power and energy demand needed to 327 meet the instantaneous speed of vehicle are calculated based 328 on a longitudinal model for the dynamics of the vehicle, as 329 given in (2) and (3) [31]. The power demand is calculated by 330 integrating net forces acting in the direction of motion over 331 time. The acting forces are the aerodynamic drag $F_{D}$, the time 332 derivative of momentum in the moving direction $m d v / d t$, the 333 
TABLE II

VAlues of PARAmeters USED IN POWER AND ENERGy CALCUlation

\begin{tabular}{ccccccr}
\hline \hline Parameter & $\rho$ & $A_{f}$ & $C_{D}$ & $V_{W}$ & $m$ & $\theta$ \\
Value & $1.2 \mathrm{~kg} / \mathrm{m}^{3}$ & $2.5 \mathrm{~m}^{2}$ & 0.3 & $0 \mathrm{~m} / \mathrm{s}$ & $1550 \mathrm{~kg}$ & 0 \\
\hline \hline
\end{tabular}

334 rolling friction $F_{F}$, and the road grade force $F_{G}$ [32], [33]. The 335 governing equations are given as follows:

$$
\begin{aligned}
& P=\left(m \frac{d v}{d t}+F_{D}+F_{F}+F_{G}\right) v \\
& E=\int_{0}^{t}\left(m \frac{d v}{d t}+F_{D}+F_{F}+F_{G}\right) \cdot v \cdot d t
\end{aligned}
$$

336 where

$$
\begin{aligned}
& F_{D}=\rho A_{f} C_{D}\left(v+v_{w}\right)^{2} / 2 \\
& F_{F}=(1+v / 100) \cdot m g \cdot \cos \theta / 100 \\
& F_{G}=m g \sin \theta
\end{aligned}
$$

$337 \rho$ is the air density, $A_{f}$ is the vehicle frontal area, $C_{D}$ is 338 the aerodynamic drag coefficient, $V_{W}$ is the head-wind speed, $339 \mathrm{~m}$ is the mass of the vehicle, $g$ is the gravitational constant $340\left(9.8 \mathrm{~m} / \mathrm{s}^{2}\right)$, and $\theta$ is the grade angle of the road. In this paper, 341 typical values for a midsize sedan, as given in Table II, are used 342 in the energy and power calculations.

343 This assumption is in line with the dominance of sedans in 344 urban transportation fleet, which is also mirrored in the fleet of 345 participant vehicles in the data-collection phase. Positive values 346 of power demand indicate the power to be provided by the 347 propulsion system at the wheels. The total daily energy demand 348 is calculated by integrating the positive values of the power 349 demand over time divided by the number of data-collection 350 days. Negative values of power demand represent the power 351 to be dissipated as heat by conventional breaking systems or 352 partially recovered in regenerative breaking systems.

\section{B. Enhancement of the Candidate Driving Cycles}

354 It is clear that the two candidate driving cycles do not 355 necessarily match all the average values uniformly well, al356 though they have the best figures of merit for single daily 357 cycles in the database. In this paper, for instance, figures of 358 merit for weekday and weekend candidate cycles are 0.197 359 and 0.295 , respectively. To enhance the quality of the candidate 360 cycles, further processing is done with a view to improve their 361 figure of merit using microtrips of other cycles available in 362 the database. The candidate cycles are then enhanced based 363 on a methodology shown in Fig. 3. A maximum 5\% deviation 364 from average daily energy demand for final driving cycles is 365 allowed in the construction of the enhanced candidate cycles. 366 The average values for the enhanced candidate cycles are also 367 given in Table I.

368 The recorded speed-time traces from the database are split 369 into "microtrips." A microtrip is defined as a snippet of the 370 speed-time trace that begins and ends at idle states: zero speed. 371 The microtrips are then classified according to their traffic

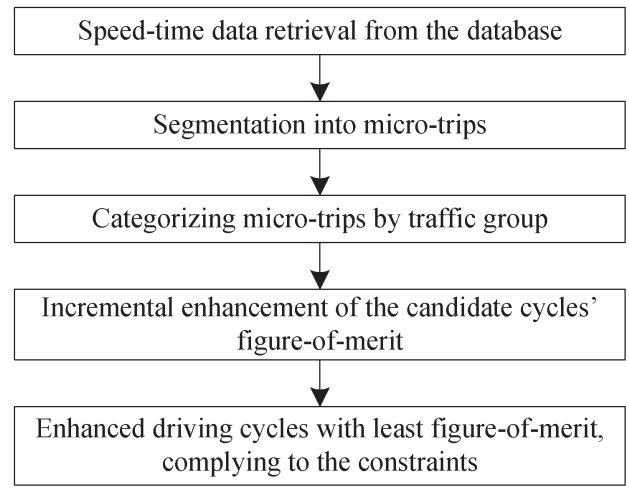

Fig. 3. Methodology of enhancing the candidate cycles.

TABLE III

MICROTRIP CLUSTERING CRITERIA

\begin{tabular}{lll}
\hline \hline Traffic category & Average speed & \multicolumn{1}{c}{ Acceleration } \\
\hline Congested & Low: $[05] \mathrm{km} / \mathrm{h}$ & Mild: $[-0.1,0.1] \mathrm{m} / \mathrm{s}^{2}$ \\
Urban & Moderate: $[5,40] \mathrm{km} / \mathrm{h}$ & Harsh: $[-3.0,3.0] \mathrm{m} / \mathrm{s}^{2}$ \\
Highway & High: $[40100] \mathrm{km} / \mathrm{h}$ & Moderate: $[-1.0,1.0] \mathrm{m} / \mathrm{s}^{2}$ \\
\hline \hline
\end{tabular}

groups characterized by average speed and acceleration, as 372 given in Table III. Here, each microtrip of the candidate cycle is 373 iteratively exchanged with microtrips of the same traffic group 374 until the best figure of merit $\sigma$ is obtained.

375

Classification of microtrips is an important step in the 376 cycle-enhancement method that describes their physical char- 377 acteristics in terms of driving patterns and traffic conditions. 378 Congested traffic such as stop-and-go patterns is characterized 379 by low average speed and mild acceleration (e.g., driving pat- 380 tern in main commuting streets during rush hours). Urban traffic 381 is designated by its moderate average speed and wider range 382 of acceleration typically governed by stop signs and traffic 383 lights in normal urban driving. Finally, the distinct feature of 384 highway traffic is high average speed and moderate acceleration 385 rates. The variations in the speed and acceleration can change 386 the power demand accordingly, and hence, the time percent- 387 ages spent in various speed and acceleration ranges provide 388 important information about power demand in different traffic 389 categories defined in Table III. $\quad 390$

Speed-acceleration frequency distribution (SAFD) plots pro- 391 vide the needed information about the time proportions of 392 individual driving modes [34]. The use of microtrips of the 393 same traffic group serves to maintain the matching of the SAFD 394 of the two candidate cycles to that of the SAFD of all recorded 395 cycles.

An alternative approach to developing a driving cycle is to 397 use random selection methods to select the appropriate number 398 of classified microtrips required to construct a representative 399 cycle matching well with the average characterizing parame- 400 ters with the lowest figure of merit. Random combination of 401 microtrips has been used by other researchers as a means to 402 construct representative driving cycles [35]. Another approach 403 to improve the current methodology would be to give a weight- 404 ing factor to the terms in the definition of the figure of merit [see 405 (1)] to adjust sensitivity of the final result to bias characterizing 406 parameters in accordance with their importance in the final 407 

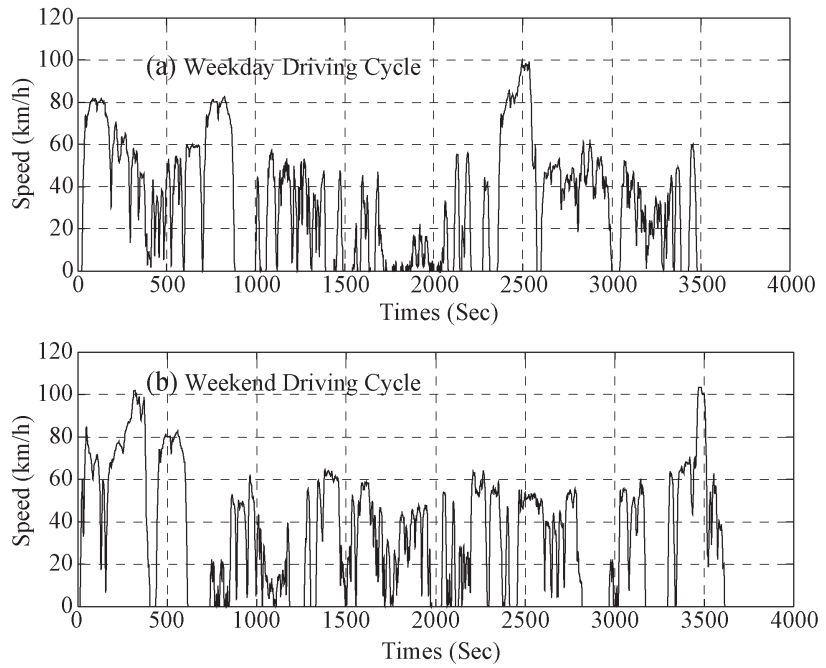

Fig. 4. Enhanced candidate driving cycles. (Top) Weekday. (Bottom) Weekend.

408 duty cycle. In this paper, all weighting factors are considered 409 equal to 1 . Evidently, energy needed and power demand for 410 traveling the same distance in different traffic modes are not 411 equal. It is also well understood that the aggressiveness of the 412 driver in accelerating and decelerating the vehicle increases 413 power consumption. However, it should be noted that replacing 414 microtrips of the candidate cycle with microtrips of the same 415 traffic mode, but potentially from different driving styles, is 416 not misleading from an energy perspective. This is due to the 417 fact that all parameters defining aggressiveness, energy level, 418 and power consumption are already included in the 25 char419 acterizing parameters used in this study, and the replacements 420 increasing the figure of merit to larger values are not con421 sidered. Implementing alternative enhancement methodologies 422 mentioned earlier and their performance assessment are left for 423 further work. Fig. 4 shows the enhanced weekday and weekend 424 candidate driving cycles. The metric units are used throughout 425 the study.

426 The speed in the driving cycles shown in Fig. 4 is given 427 in kilometers per hour; however, for more convenience, both 428 English and metric versions of the driving cycles in digital 429 format are made available to the public [36].

430 Durations of the weekday and weekend cycles are 3484 and $4313616 \mathrm{~s}$, respectively. The maximum velocity is higher in the 432 weekend cycle, i.e., $114 \mathrm{~km} / \mathrm{h}$, whereas in the weekday cycle, 433 the maximum velocity is $89.6 \mathrm{~km} / \mathrm{h}$. The enhancement process 434 does not necessarily finish by yielding a figure of merit equal to 435 zero, but a considerable improvement can be expected as, in this 436 study, the initial values were improved by approximately $40 \%$. 437 The figure of merit for the enhanced weekday and weekend 438 driving cycles are 0.15 and 0.2 , respectively. Fig. 5 shows 439 the SAFD plot for weekday and weekend enhanced candidate 440 driving cycles.

441 The two patterns are different in nature. Stop-and-go events 442 characterized by larger acceleration or deceleration rates at low 443 speeds are more probable in the weekday pattern. However, 444 high-speed events are more probable in the weekend pattern. 445 The driving pattern on the weekend is slightly more aggressive
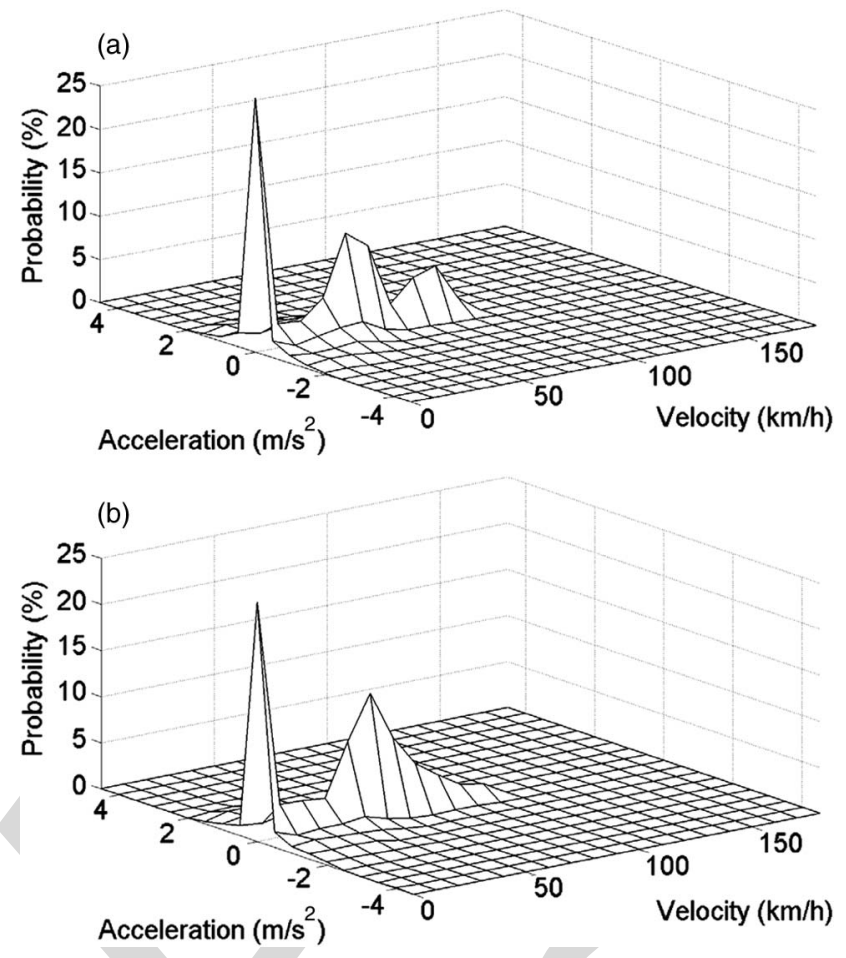

Fig. 5. SAFD plots for the enhanced candidate driving cycles. (a) Weekday. (b) Weekend.
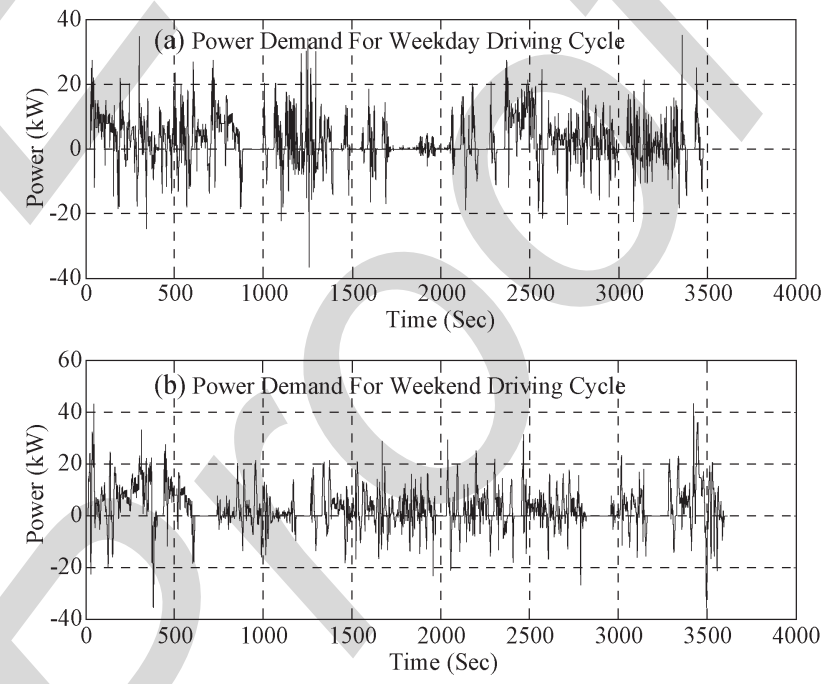

Fig. 6. Power-time traces for the enhanced candidate driving cycles. (a) Weekday. (b) Weekend.

due to higher acceleration and deceleration rates, which results 446 in higher power demand for weekend driving patterns.

The power-time profiles corresponding to the two driving 448 cycles are also presented in Fig. 6. To obtain these profiles, a 449 vehicle with the specifications listed in Table II is considered 450 to have driven the two cycles. Evidently, variations and abrupt 451 changes in power demand are more considerable in the stop- 452 and-go driving mode that is dominant in congested and urban 453 traffics in the weekday driving cycle. This mode of driving 454 considerably contributes to air pollution in downtown areas of 455 large metropolitan areas, and it is particularly important to be 456 covered by electric propulsion in the case of HEVs. 


\section{PARKING ANALYSIS}

459 Vehicle parking at home, the workplace, commercial lo460 cations, and on the street constitutes a critically important 461 element of a modern duty cycle that can address a multitude 462 of drive-train topologies, storage technologies, and controllers. 463 Developing models to analyze the parking behavior in an urban 464 area for city planning may require detailed information on the 465 parameters affecting parking behavior during the day, such as 466 travel demands, district-based knowledge on cost of parking, 467 nature of activities in the area of interest, and supply and 468 demand on an hourly basis. However, from the charging per469 spective only, relatively long parking times provide potential 470 charging times to increase the SOC of an onboard energy 471 storage device. A typical duration for a full charge under normal 472 charging conditions ( $110 \mathrm{~V}$ and $15 \mathrm{~A}$ ) for current competitive 473 battery technologies used in electric vehicles, namely, lithium 474 ion and nickel metal hydride, is approximately $6 \mathrm{~h}$; the mini475 mum duration for partial charging is presently not known with 476 a high confidence level. Therefore, it is expected that most 477 plug-in electric vehicles will be charged mainly overnight. If 478 a relatively long parking time (e.g., more than $3 \mathrm{~h}$ ) during the 479 day is considered as a realistic scenario for a reasonable share 480 of the urban fleet, it is possible to downsize the battery storage 481 capacity and reduce the capital cost of a plug-in vehicle or, 482 in the case of a PHEV with a fixed size of battery storage, 483 drive more miles on electricity to improve cost effectiveness. 484 In addition, fast charging schemes using level $2(120 \mathrm{~V}$ and $48530 \mathrm{~A})$ and direct dc chargers will facilitate full charging in 486 shorter charging durations, i.e., as low as $20 \mathrm{~min}$, depending 487 on the battery technology and dc charging infrastructure.

488 It is also important to highlight that identification of parking 489 locations for charging opportunities is critical; this aspect has 490 been of strong interest to utilities, as they need to address both 491 power and energy demand for electric mobility, with power 492 having a large impact on grid infrastructure costs. Charging 493 at residential areas, parking lots at work places, and large 494 shopping malls is of interest; however, parking in streets or 495 less-frequently open areas is not emphasized due to the large 496 infrastructure cost required to achieve this type of opportunity 497 charging. In the development of a duty cycle in this work, 498 parking events are described for all categories over a 24-h 499 period to provide data for any combination of future charging 500 scenarios. Inherent in this study is the assumption that plug501 in vehicle owners will not be significantly modifying their 502 parking behaviors, although they may change their parking stall 503 to access a plug.

504 An extensive literature survey reveals that a few studies 505 have focused on the potential charging aspect of parking times 506 as a part of daily activities of vehicles; none seem to have 507 incorporated this into a duty cycle. This aspect is of critical 508 importance to address energy drivers in transportation and 509 allow the displacement of fossil fuels with new renewable 510 energy generation. The analysis conducted by the Argonne 511 National Laboratory (ANL) shows that, in the United States, $51266 \%$ of the vehicles driven to work are parked more than $5133 \mathrm{~h}$ before noon, potentially allowing a second charge before 514 the electric utility peak demand begins [37]. It was also ob515 served that vehicles were parked for a short time for shop-
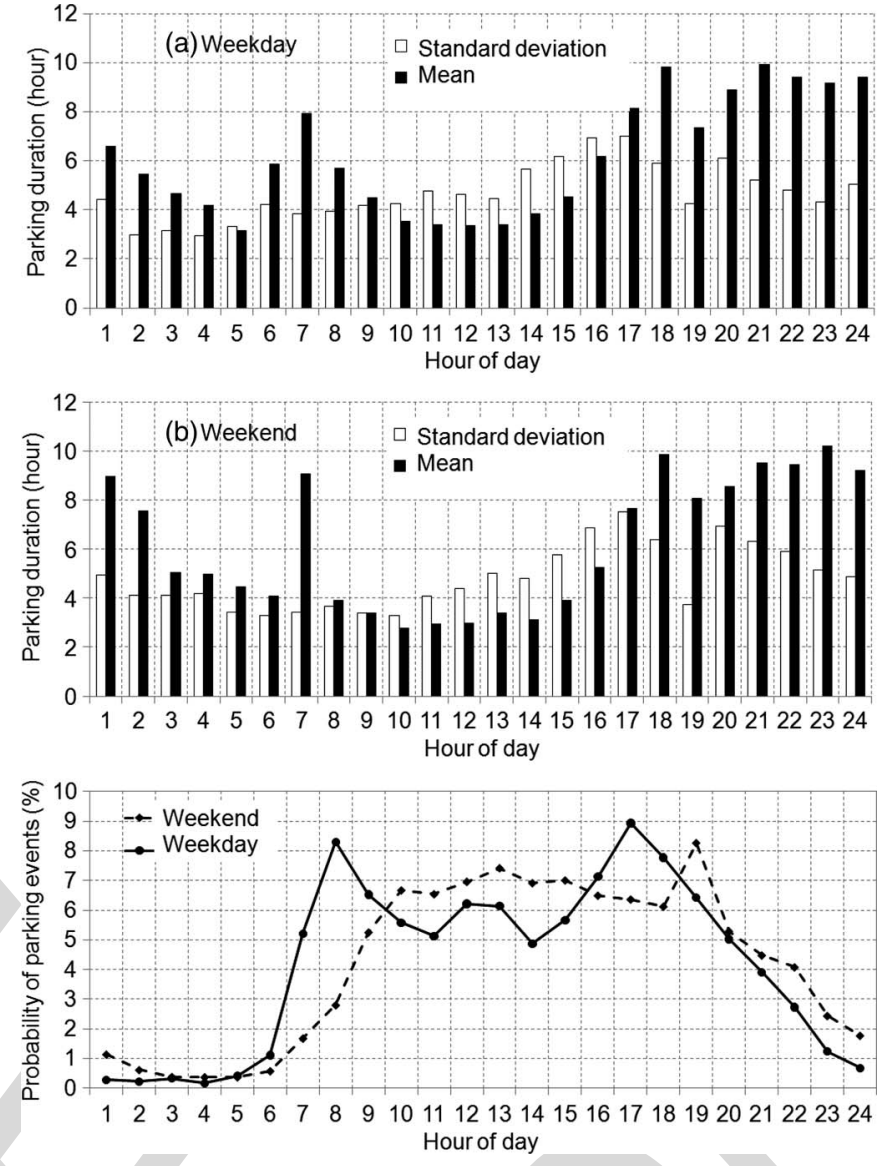

Fig. 7. Mean and standard deviation of parking duration by hour of day. (a) Weekdays. (b) Weekends. (c) Probability of parking events by hour of day for both weekdays and weekends.

ping purposes, and parking was often during peak electric 516 demand [36].

In this paper, GPS-based data loggers provide information 518 on location, type of parking, and duration of parking events. 519 The type of parking can be determined from the driver's 520 behavior, where it is relatively simple to determine where 521 the person lives and works; commercial parking locations 522 are found by digitizing commercial parking lots in Winnipeg. 523 Street parking is deduced from a car staying on the street. Note 524 that studies dedicated to record the instantaneous speed of the 525 vehicle for certification purposes, such as that leading to the 526 FTP72 standard cycle and its enhanced cycles, do not provide 527 information on parking times. Here, probable parking times 528 and average duration for each parking event, as well as the 529 standard deviation of the data points, are included to adequately 530 reflect a daily driving and parking profile for electric vehicle 531 design of drive-train topologies.

Parking times of less than half an hour are arbitrarily clas- 533 sified in our study as short; the distribution of such parking 534 events shows that, in early mornings and afternoons, this type 535 of parking is the most likely. It is important to note that stop 536 times of less than $2 \mathrm{~min}$, happening at stop signs or traffic 537 lights, are excluded from short parking. The results of short 538 parking times are not presented here as it is assumed that, in 539 the real world, these occasional parking events are not favored 540 by drivers for charging. However, a cumulative parking time 541 
542 representing short parking times per day will be included in the 543 final duty cycles. The results of studying parking periods that 544 exceed 30 min are shown in Fig. 7.

545 Fig. 7(a) and (b) shows mean values and standard deviations 546 of parking durations by hour of day for weekdays and week547 ends, respectively.

548 Two distinct patterns in daily parking behavior can be recog549 nized, namely, a mean value less than or equal to the standard 550 deviation and a mean value larger than the standard deviation. 551 Long parking duration with a mean value larger than the stan552 dard deviation happens between 8 P.M. and 6 A.M., showing 553 that the majority of drivers tend to park their vehicles for a long 554 time. However, when the standard deviation is greater than the 555 mean value of parking duration, the data show an increase in 556 daily trips, happening between 10 A.M. and 4 P.M., and the 557 average of the mean values is about $4 \mathrm{~h}$. Peaks in probability 558 of parking events in terms of hour of day, as shown in Fig. 7(c), 559 reflect the difference in nature of activities between weekdays 560 and weekends. While there is only one peak in the weekend 561 curve happening at 7 P.M., there are three peaks occurring at 562 7A.M., 12 P.M., and 5 P.M. during weekdays.

563 On weekdays, $67 \%$ of the vehicles park for more than $3 \mathrm{~h}$ be564 tween 6 A.M. and 9 A.M., whereas between 9 A.M. and 12 P.M., 565 only $33 \%$ of the vehicles park for more than $3 \mathrm{~h}$. This can 566 be justified as many commuters drive to work and park their 567 car during working hours at their working place early in the 568 morning. However, after 9 A.M., vehicles moving in the streets 569 tend to park for a limited duration, i.e., typically less than $3 \mathrm{~h}$, 570 which is necessary for activities such as shopping. The analysis 571 shows that, while overnight charging is the first choice for 572 charging the battery, second charging is most likely to happen 573 in the morning around 9 A.M. or in the afternoon around 5 P.M. 574 Taking the higher electricity price in peak hours in many 575 jurisdictions, a more realistic scenario for charging would be 576 overnight followed by early mornings. However, the distribu577 tion of charging load on the electric grid overnight or during the 578 day would be different, which indicates a need to redesign the 579 location of feeders in the city. This aspect of charging electric 580 cars is beyond the scope of this study and will be published in 581 separate articles of various charging opportunities and power 582 levels.

\section{Assembly of the Driving CyCles}

584 Analysis was performed on the raw GPS data in conjunction 585 with the digitized maps of the roads and commercial parking 586 locations to characterize the driving and parking behavior of 587 the vehicles under the one-year span of the survey in the 588 city of Winnipeg. Using the method shown in Fig. 2, two 589 24-h vehicle usage profiles representing a daily duty cycle 590 were developed for both weekdays and weekends. The parking 591 patterns obtained from the analysis of parking times, as well as 592 cumulative short parking events, are included in the daily duty 593 cycles for weekdays and weekends. In creating this, the average 594 distance traveled in driving events is considered to separate 595 the final driving cycle into parts, and then, parking events are 596 inserted in between in the most probable way. The resulting 597 cycles are shown in Fig. 8 and are meant to represent the
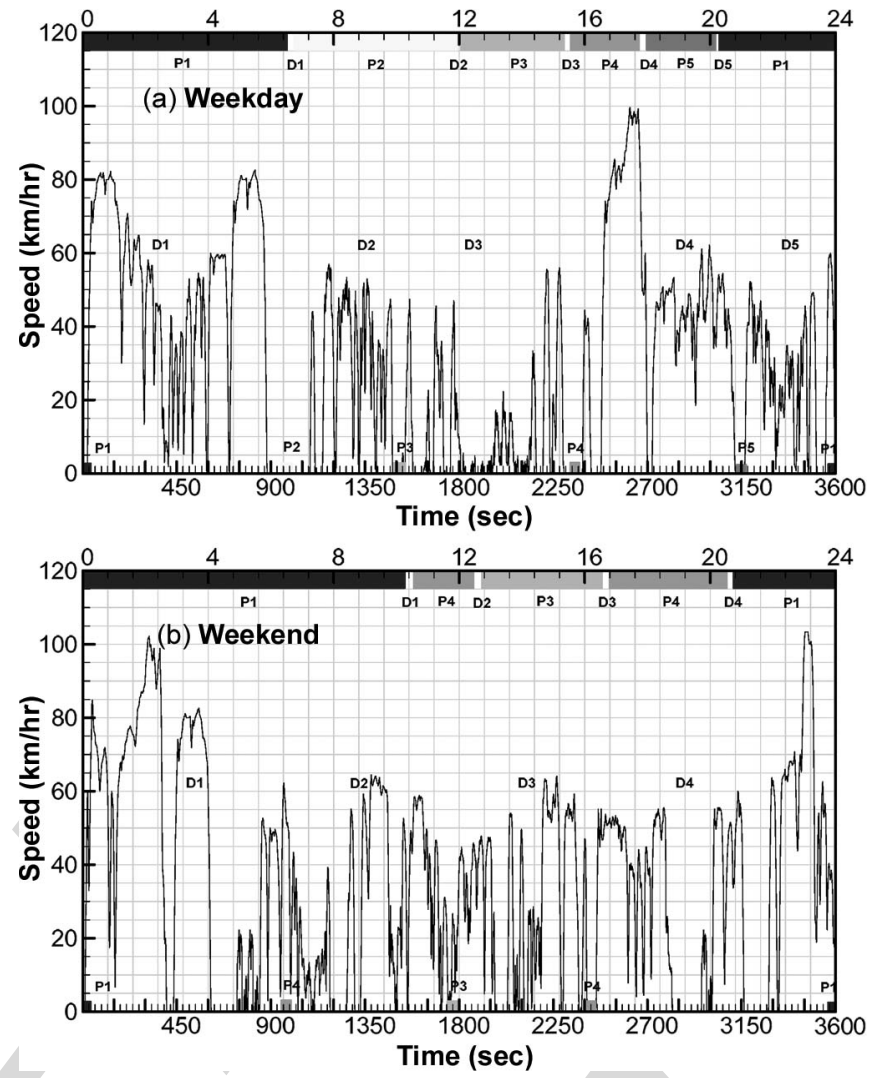

Fig. 8. Resulting 24-h duty cycles. (a) Weekdays. (b) Weekends.

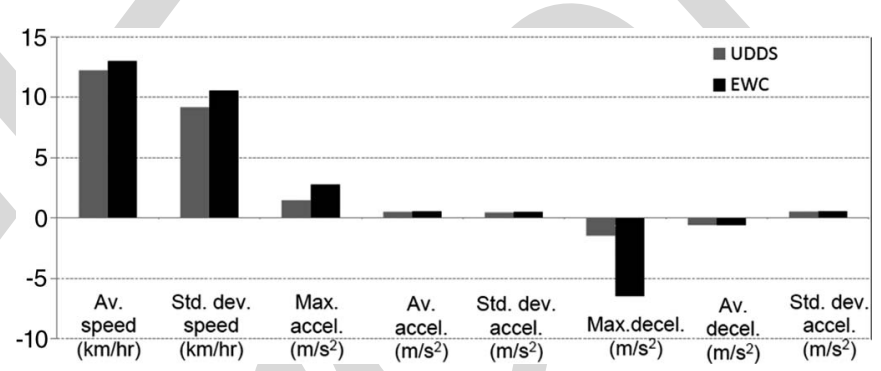

Fig. 9. Comparison between the standard cycle UDDS and the enhanced weekday cycle (EWC)

44 million data points into a condensed duty cycle for studies 598 pertaining to plug-in hybrids, including optimization of power 599 trains [29]. In Fig. 8, D stands for driving, P stands for parking 600 periods, P1 stands for home, P2 stands for work, P3 stands 601 for commercial, P4 stands for short stops, and P5 stands for 602 street parking. The driving cycles are on a 1-h basis, and the 603 duty cycles, with long parking times included, are on a 24-h 604 basis and are both combined into the same figure. The parking 605 durations on a 24-h scale designated by color codes are also 606 shown on a 1-h driving scale for the sake of clarity. The parking 607 events that potentially can be used for charging are P1, P2, or P3 608 when the vehicle is most probably parked in a parking spot with 609 access to level-1 or level-2 charging. The parking events that 610 happen on the street or short parking durations are considered 611 not suitable for charging.

612

Some characteristics of the enhanced driving cycle are com- 613 pared with those of the standard cycle UDDS, and the results 614 are presented in Fig. 9. The comparison indicates that more 615 aggressive characteristics are associated with the real-world 616 
617 cycle, whereas, on average, the two cycles may be considered 618 interchangeable.

619 Evidently, the vehicles with the opportunity to charge limited 620 to overnight have more time to be fully charged under slow 621 charging mode on the weekends. This is particularly important 622 for PEVs with larger battery storage capacity. Deriving a grid 623 load based on this driving cycle has merit but requires the 624 understanding of its limitations for utilities; however, it is 625 beyond the scope of this work.

626 The data files of the duty cycles and the collected raw data 627 are available to the public on the World Wide Web through a 628 unique Digital Object Identifier number [36]. Forty-four mil629 lion speed-time data points, stamped with date and time, and 630 collected over the course of one year are made available. The 631 latitude and longitude of the position of the vehicles recorded 632 on a secondly basis are masked by mapping the starting point of 633 every trip to $(0,0)$ to respect the confidentiality agreement with 634 the participants in the data-collection phase. However, personal 635 information about the participants is used to label the parking 636 locations as home or work. Labeled parking locations, as well 637 as the duration of parking events needed for further analyses 638 with different charging scenarios in the case of plug-in electric 639 vehicles, are included in the data files. The parking events that 640 are less than $30 \mathrm{~min}$ in duration are labeled short stops. Parking 641 events happening along the street are not potentially suitable 642 for charging. The locations of the parking events longer than $64330 \mathrm{~min}$ in duration are marked home, work, shop, and street. The 644 effectiveness of the methodology presented in this study, even 645 with far fewer data points (about 1 million data points, which 646 is equal to about $2 \%$ of the data points used in this study), 647 for simulation-based optimization of a PHEV was shown in [29].

\section{CONCLUSION}

649 A new approach to the development of a duty cycle that ad650 dresses the requirements associated with the design of electric 651 vehicles-e.g., HEV, PHEV, BEV, and extended-range vehi652 cles, has been proposed and implemented on a $24-\mathrm{h}$ timescale. 653 It provides a complete data set for optimization of battery 654 size for on-road vehicles in a typical North American urban 655 setting. For example, power and energy demand in the daily 656 operation of a sedan is directly related to the rate of acceleration 657 and deceleration and time spent in different traffic modes; 658 charging scenarios depend on parking times and duration. The 659 driving behavior of a fleet of 76 participants in a one-year 660 voluntary data-collection program in the city of Winnipeg is 661 analyzed to develop a driving cycle and is composed of two 662 24-h duty cycles for weekdays and weekends. This cycle pro663 vides information about the time and duration of driving in 664 different traffic categories, as well as information on parking 665 times when the vehicle is not in use. Further vehicle simu666 lation tools can use the daily duty cycles developed to op667 timally design propulsion systems, drive-train configurations, 668 and storage components for PEV technologies under real-world 669 driving conditions. Furthermore, this information can be used 670 to analyze the impact of daytime charging by a fleet of plug671 in electric vehicles on the electric utility grid that may create a 672 peak demand during the day to be met by the local utility grid.
The target use of the developed cycle is to provide a duty cycle 673 that can be used to optimally address energy drivers simultane- 674 ously facing transportation by displacing fossil fuels with new 675 renewable energy generations with the direct consequences of 676 increasing the renewable energy ratio of various jurisdictions. 677

To achieve this goal, 25 parameters characterizing a driving 678 cycle for further PEV simulations are recognized, and two 679 candidate daily cycles having the closest match to the average 680 of the parameters are selected. The candidate cycles are then 681 incrementally enhanced by replacing their microtrips with those 682 extracted from the same traffic group, minimizing a figure of 683 merit defined based on the characterizing parameters. Finally, 684 the processed parking data are included to complete two 24-h 685 duty cycles. The final result is therefore reflecting more accu- 686 rately a realistic driving pattern than driving cycles resulting 687 from methodologies that patch snippets of driving data from 688 different drivers or occasions to make a driving cycle. Although 689 the data collected represent driving behavior in the city of 690 Winnipeg, MB, Canada, the methodology presented here can 691 be extended to any other urban area of interest.

692

There exist a few directions to continue the research on or 693 using the collected data. Other methods for development of a 694 driving cycle may include a stochastic approach for selecting 695 and patching snippets of speed-time traces using a probability 696 matrix [14], [21], which might be enhanced to incorporate 697 power and energy requirements of the vehicle. Future work 698 may also concentrate on one specific driving pattern, for in- 699 stance, a commuter, to develop a dedicated driving cycle best 700 mirroring that particular driving pattern. The driving cycle may 701 also be used for a wide range of applications, such as energy 702 assessment of the vehicles in daily use in urban transportation, 703 analysis of charging scenarios in PHEVs and PEVs, vehicle-to- 704 grid analysis, and statistical assessment of driving cycle vari- 705 ability on hybrid drive-train design. Finding other applications, 706 particularly from a social driving behavior perspective, can also 707 be viewed as an important extension of the work.

708

\section{ACKNOWLEDGMENT}

709

The authors would like to thank Prof. D. Blair and R. Smith 710 from the Department of Geography, University of Winnipeg, for 711 providing the collected travel data. Ongoing discussions with 712 Emerging Energy Systems at Manitoba Hydro, in particular 713 with T. Molinski, are acknowledged. Special thanks to Presen- 714 tech Inc. for integrating the parking study with their proprietary 715 firmware.

\section{REFERENCES}

[1] E. Ungar and K. Fell, "Plug in, turn on, and load up," IEEE Power Energy 718 Mag., vol. 8, no. 3, pp. 30-35, May/Jun. 2010.

[2] J. Tollefson, "Charging up the future," Nature, vol. 456, no. 7221, 720 pp. 436-440, Nov. $27,2008$.

[3] A. A. Frank, "Plug-in hybrid vehicles for a sustainable future," Amer. Sci., 722 vol. 95 , no. 2, pp. 158-165, Mar./Apr. 2007.

[4] T. H. Bradley and A. A. Frank, "Design, demonstrations and sustainability 724 impact assessments for plug-in hybrid electric vehicles," Renew. Sustain. 725 Energy Rev., vol. 13, no. 1, pp. 115-128, Jan. 2009.

[5] C. N. Shiau, C. Samaras, R. Hauffe, and J. J. Michalek, "Impact of battery 727 weight and charging patterns on the economic and environmental benefits 728 of plug-in hybrid vehicles," Energy Policy, vol. 37, no. 7, pp. 2653-2663, 729 Jul. 2009. 
[6] A. F. Burke, "Batteries and ultra capacitors for electric, hybrid and fuel cell vehicles," Proc. IEEE, vol. 95, no. 4, pp. 806-820, Apr. 2007.

7] J. J. Michalek, P. Y. Papalambros, and S. J. Skerlos, "A study of fuel efficiency and emission policy impact on optimal vehicle design decisions," ASME J. Mech. Des., vol. 126, no. 6, pp. 1062-1070, Nov. 2004

8] M. Rahimi and A. Emadi, "An analytical investigation of DC/DC power electronic converters with constant power loads in vehicular power systems," IEEE Trans. Veh. Technol., vol. 58, no. 6, pp. 2689-2702, Jul. 2009.

[9] A. Emadi, M. Ehsani, and J. M. Miller, Vehicular Electric Power Systems: Land, Sea, Air, and Space Vehicles. New York: Marcel Dekker, Dec. 2003

0] M. Ehsani and A. Emadi, "Multiconverter power systems and their applications," J. Elect. Power Compon. Syst., vol. 29, no. 10, pp. 949-963, Oct. 2001.

1] A. Emadi, A. Khaligh, C. H. Rivetta, and G. A. Williamson, "Constant power loads and negative impedance instability in automotive systems: Definition, modeling, stability, and control of power electronic converters and motor drives," IEEE Trans. Veh. Technol., vol. 55, no. 4, pp. 1112 1125, Jul. 2006

2] Federal Test Procedure Review Project: Preliminary Tech. Rep. [Online]. Available: http://www.epa.gov/oms/regs/ld-hwy/ftp-rev/ftp-tech.pdf

3] T. C. Austin, F. J. DiGenova, T. R. Carlson, R. W. Joy, K. A. Gianolini, and J. M. Lee, "Characterization of driving patterns and emissions from lightduty vehicles in California," Calif. Air Resources Board, Sacramento, CA, Final Rep. Contract No. A932-185, 1993.

4] J. Lin and D. A. Neimer, "An exploratory analysis comparing a stochastic driving cycle to California's regulatory cycle," Atmos. Environ., vol. 36, no. 38, pp. 5759-5770, Dec. 2002.

5] S. H. Kamble, T. V. Mathew, and G. L. Sharma, "Development of realworld driving cycle: Case study of Pune, India," Transp. Res. Part D, vol. 14, no. 2, pp. 132-140, Mar. 2009.

6] G. Fontaras, P. Pistikopoulos, and Z. Samaras, "Experimental evaluation of hybrid vehicle fuel economy and pollutant emissions over real-world simulation driving cycles," Atmos. Environ., vol. 42, no. 18, pp. 4023 4035, Jun. 2008

7] W. T. Hung, K. M. Tam, C. P. Lee, L. Y. Chan, and C. S. Cheung, "Comparison of driving characteristics in cities of Pearl River Delta, China," Atmos. Environ., vol. 39, no. 4, pp. 615-625, Feb. 2005.

8] G. H. Tzeng and J. J. Chen, "Developing a Taipei motorcycle driving cycle for emissions and fuel economy," Transp. Res. Part D, vol. 3, no. 1, pp. 19-27, Jan. 1998.

9] A. Esteves-Booth, T. Muneer, H. Kirby, J. Kubie, and J. Hunter, "The measurement of vehicular driving cycle within the city of Edinburgh," Transp. Res. Part D, vol. 6, no. 3, pp. 209-220, May 2001.

[20] M. Andre, "Driving cycle development: Characterization of the methods," in Proc. SAE Int. Spring Fuels Lubricants Meet., 1996, pp. 1-13, Technical Paper Series 961112 .

1] J. Lin and D. A. Neimer, "Estimating regional air quality vehicle emission inventories: Constructing robust driving cycles," Transp. Sci., vol. 37, no. 3, pp. 330-346, Aug. 2003.

22] J. Gonder, T. Markel, M. Tornton, and A. Simpson, "Using global positioning system travel data to access real world energy use of plug in hybrid electric vehicles," Transp. Res. Rec., no. 2017, pp. 26-32, Washington, DC, 2007.

3] B. Liaw and M. Dubarry, "From driving cycle analysis to understanding battery performance in real-life electric hybrid vehicle operation," J. Power Sources, vol. 174, no. 1, pp. 76-88, Nov. 2007.

24] A. Fröberg and L. Nielsen, "Efficient drive cycle simulation," IEEE Trans. Veh. Technol., vol. 57, no. 3, pp. 1442-1453, May 2008.

25] Q. Gong, Y. Li, and Z. Peng, "Trip-based optimal power management of plug-in hybrid electric vehicles," IEEE Trans. Veh. Technol., vol. 57, no. 6, pp. 3393-3401, Nov. 2008.

26] J. P. Markey, "Findings from EPA's study of in-use driving patterns," in Proc. 3rd Annu. CRC-APRAC On-Road Veh. Emissions Workshop, San Diego, CA, 1992.

27] E. Milkins and H. Watson, "Comparison of urban driving patterns," presented at the Soc. Automotive Eng. Conf., Warrendale, PA, 1983, Paper 830939.

28] J. E. Morey, T. Limanond, and D. A. Niemeier, "Validity of chase car data used in developing emissions cycles," J. Transp. Stat., vol. 3, no. 2, pp. 15-28, 2000.

29] E. Tara, S. Shahidinejad, S. Filizadeh, and E. Bibeau, "Battery storage sizing in a retrofitted plug-in hybrid electric vehicle," IEEE Trans. Veh. Technol., to be published.
[30] A. A. Pesaran, T. Markel, H. S. Tataria, and D. Howell, "Battery require- 808 ments for plug-in hybrid electric vehicles-Analysis and rationale," in 809 Proc. 23rd Int. EVS, Anaheim, CA, 2007.

[31] J. Y. Wong, Theory of Ground Vehicles. New York: Wiley-Interscience, 811 2001.

[32] T. D. Gillespie, Fundamentals of Vehicle Dynamics. Warrendale, PA: 813 SAE, 1992.

[33] M. Ehsani, Y. Gao, and A. Emadi, Modern Electric, Hybrid Electric, and 815 Fuel Cell Vehicles: Fundamentals, Theory, and Design. Boca Raton, FL: 816 CRC, 2004.

[34] E. Milkins and H. Watson, "Comparison of urban driving patterns, motor 818 vehicle technology: Progress and harmony," in Proc. 2nd Int. Pacific Conf. 819 Autom. Eng., Tokyo, Japan, 1983, pp. 735-746.

[35] T. C. Austin, F. J. DiGenova, T. R. Carlson, R. W. Joy, K. A. Gianolini, 821 and J. M. Lee, Characterization of Driving Patterns and Emissions From 822 Light-Duty Vehicles in California. Sacramento, CA: Sierra Res., 1993. 823 (Prepared for California Air Resources Board).

[36] S. Shahidinejad, E. L. Bibeau, and S. Filizadeh, Winnipeg Driving Cycle: 825 WPG02. DOI:10.5203/ds_bib_1. [Online]. Available: http://mspace.lib. 826 umanitoba.ca/dspace/handle/1993/3997

[37] Argonne Nat. Lab., Transportation Research Development. [Online]. 828 Available: http://www.transportation.anl.gov/pdfs/HV/548.pdf 829

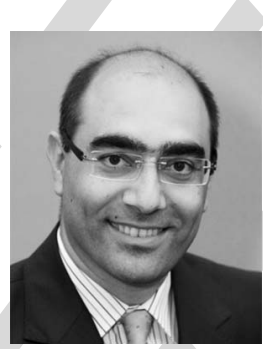

Soheil Shahidinejad received the B.Sc., M.Sc., and 830 $\mathrm{Ph} . \mathrm{D}$. (with honors) degrees from Sharif University 831 of Technology, Tehran, Iran, in 1993, 1996 and 2002, 832 respectively, all in mechanical engineering. $\quad 833$

$\mathrm{He}$ is currently a Research Associate with the 834 Department of Mechanical and Manufacturing 835 Engineering, University of Manitoba, Winnipeg, 836 MB, Canada. His research deals with the study and 837 analysis of energy-efficient systems and renewable 838 energies.

Automotive Engineers. He was a recipient of a one-year scholarship in 84 1999/2000 from the French Government to extend his Ph.D. research with the 842 Institut National Polytechnique de Lorraine, Nancy, France.

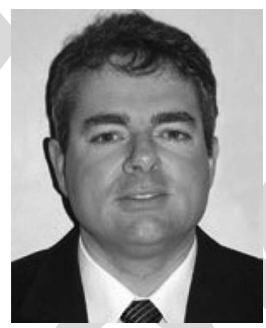

Eric Bibeau received the B.Sc. degree from McGill 844 University, Montreal, QC, Canada, in 1986 and the 845 M.Sc. and Ph.D. degrees from the University of 846 British Columbia, Vancouver, BC, Canada, in 1988847 and 1993, respectively.

848

He currently is an Associate Professor with the 849 University of Manitoba, Winnipeg, MB, Canada, 850 where he holds the National Science and Engineering 851 Research Council of Canada/Manitoba Hydro Chair 852 in Alternative Energy. He specializes in developing 853 power systems using biomass feedstocks, industrial 854 waste heat, and kinetic energy from rivers for distributed energy applications. 855 $\mathrm{He}$ is also involved in developing blade-deicing-mitigation strategies for wind 856 turbines. He is developing expertise in electric vehicles and district energy 857 systems as a means to increase the renewable energy ratio in Canada to 858 simultaneously address climate change and peak oil energy drivers.

Dr. Bibeau is a Registered Professional Engineer in the Province of 860 Manitoba.

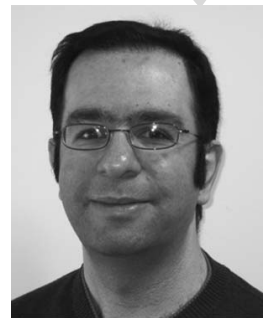

Shaahin Filizadeh (S'02-M'04) received the B.Sc. 862 and M.Sc. degrees in electrical engineering from 863 Sharif University of Technology, Tehran, Iran, in 864 1996 and 1998, respectively, and the Ph.D. degree 865 in electrical engineering from the University of 866 Manitoba, Winnipeg, MB, Canada, in 2004.

$\mathrm{He}$ is currently an Associate Professor with the 868 Department of Electrical and Computer Engineering, 869 University of Manitoba. His areas of interest include 870 power systems electromagnetic transient simulation, 871 nonlinear optimization, and power-electronic appli- 872 cations in power systems and vehicle propulsion.

Dr. Filizadeh is a Registered Professional Engineer in the Province of 874 Manitoba. 


\section{AUTHOR QUERIES}

\section{AUTHOR PLEASE ANSWER ALL QUERIES}

AQ1 = Please provide publication update in Ref. [29].

Notes:

Note that reference [4] and [6] are the same. Therefore, reference [6] was deleted from the list. Citations were renumbered accordingly. Please check.

Note that reference [3] and [7] are the same. Therefore, reference [7] was deleted from the list. Citations were renumbered accordingly. Please check.

\section{END OF ALL QUERIES}

